\title{
镍促进电化学还原交叉偶联合成偕二氟烯烃
}

\author{
常哲王佳金金陆 喜* 傅 尧* \\ (中国科学技术大学应用化学系 合肥 230026)
}

\begin{abstract}
摘要 偕二氟烯烃是一类重要的含氟有机化合物, 在有机合成化学和药物化学研究领域展现出独特的结构优势. 例如, 偕二氟烯基可以便利地转化为单氟烯基、二氟烷基、三氟甲基以及其他多种含氟结构. 偕二氟烯基结构作为䍩基理想 的电子等排体在药物设计研究中也有广泛的应用. 报道了一种镍促进的电化学还原交叉偶联反应合成功能化的偕二氟 烯烃. 该反应在非分隔电解槽中进行, 在温和的电化学还原条件下, 实现了三氟甲基烯烃烯丙基脱氟、氧化还原活性羧 酸酯脱羧或者烷基卤化物脱卤素的有机结合. 反应可有效避免使用化学计量的金属粉末或有机还原剂. 该反应为含偕 二氟烯基功能结构的生物活性分子提供了有效的合成途径.
\end{abstract}

关键词＼cjkstart偕二氟烯烃; 三氟甲基烯烃; 氧化还原活性羧酸酯; 烷基卤化物; 还原交叉偶联; 电化学

\section{Synthesis of gem-Difluoroalkenes through Nickel-Promoted Electrochemical Reductive Cross-Coupling}

\author{
Chang, Zhe Wang, Jiaxin $\mathrm{Lu}, \mathrm{Xi}^{*} \quad \mathrm{Fu}, \mathrm{YaO}^{*}$ \\ (Department of Applied Chemistry, University of Science and Technology of China, Hefei 230026)
}

\begin{abstract}
Difluoroalkenes are important fluorine-containing compounds with structural superiority in organic synthetic chemistry and medicinal chemistry. For instance, the gem-difluoroethylene moiety could be converted to monofluoroalkenyl, difluoroalkyl, trifluoromethyl moiety, and many other fluorine-containing structures conveniently. The gem-difluoroethylene moiety is also an ideal carbonyl group bioisostere and has already been widely used in drug design. Herein, a nickel-promoted electrochemical reductive cross-coupling to access functionalized and substituted gem-difluoroalkenes is reported. This reaction enables the combination of allylic defluorination of trifluoromethyl alkenes and decarboxylation of redox-active esters or dehalogenation of alkyl halides under mild electrochemical reduction conditions and in an undivided cell, therefore obviates the use of stoichiometric amount metal powder or organic reductants. Furthermore, this reaction provides efficient access to biologically interesting molecules containing gem-difluoroethylene moiety.

Keywords gem-difluoroalkene; trifluoromethyl alkene; redox-active ester; alkyl halide; reductive cross-coupling; electrochemistry
\end{abstract}

\section{Introduction}

gem-Difluoroalkenes are a kind of striking fluorinecontaining compounds with structural superiority in organic synthetic chemistry and medicinal chemistry. ${ }^{[1]}$ The gem-difluoroethylene moiety could be converted to many other fluorine-containing structures conveniently (Figure 1a), ${ }^{[2]}$ such as defluorinative functionalization to access monofluoroalkenyl moiety, ${ }^{[3]}$ hydrogenation or hydrofunctionalization to access difluoroalkyl moiety, ${ }^{[4]}$ fluorination functionalization to produce trifluoromethyl moiety. ${ }^{[5]}$ The gem-difluoroethylene moiety is also an ideal carbonyl group bioisostere and has already been widely used in drug design. ${ }^{[6]}$ Direct difluoroolefination of ketones or diazo compounds was an efficient strategy to deliver gemdifluoroalkenes (Figures 1b, i) $.{ }^{[7]} \mathrm{Hu},{ }^{[8]} \mathrm{Xiao},{ }^{[9]}$ Wang, ${ }^{[10]}$ and others enjoyed outstanding achievements in this area. More recently, allylic defluorinative functionalization of trifluoromethyl alkenes with different nucleophiles has been developed as powerful tools to synthesize substituted gem-difluoroalkenes (Figures 1b, ii). ${ }^{[11]}$ For example, Cao, ${ }^{[12]} \mathrm{Shi}^{[13]}$ Murakami, ${ }^{[14]}$ Hayashi, ${ }^{[15]}$ Zhang, ${ }^{[16]}$ and others reported the defluorinative carbonation of trifluo-

\footnotetext{
* Corresponding authors. E-mail: luxi@mail.ustc.edu.cn; fuyao@ustc.edu.cn Received August 6, 2021; revised September 5, 2021; published online September 14, 2021.

Project supported by the National Natural Science Foundation of China (Nos. 21732006, 51821006, 51961135104), and the Research Funds of the Double First-Class Initiative of University of Science and Technology of China (No. YD3530002002).

国家自然科学基金(Nos. 21732006, 51821006, 51961135104)和中国科学技术大学双一流研究基金(No. YD3530002002)资助项目.
} 
romethyl alkenes with Grignard, organozinc, organoboron, and other organometallic reagents. Molander, ${ }^{[17]} \mathrm{Zhou}^{[18]}$ and others ${ }^{[19]}$ achieved the defluorinative coupling of trifluoromethyl alkenes with radical precursors under photocatalysis conditions.

In 2017, our group ${ }^{[20]}$ proposed a concept of defluorinative reductive cross-coupling, which combined the $\mathrm{C}-\mathrm{F}$ cleavage and alkyl electrophiles activation under mild reductive reaction conditions. Our group ${ }^{[21]}$ and $\mathrm{Wang}^{[22]}$ independently extended this concept to the allylic defluorinative reductive alkylation of trifluoromethyl alkenes and offered straightforward access to gem-difluoroalkenes (Figure 1c). Later, more attention has been paid to the nickel-catalyzed or transition-metal-free defluorinative reductive cross-couplings. ${ }^{[23]} \mathrm{A}$ growing number of radical precursors generated under mild reductive conditions has been applied to the defluorinative functionalization reactions, such as epoxides, Katritzky salts, and cycloketone oxime esters. ${ }^{[24]}$ These reactions exhibited high efficiency and broad functional group compatibility. However, one of the limitations was using the stoichiometric amount of zinc or bis(pinacolato)diboron reductant.

Electrochemical synthesis has a unique attraction for organic synthetic chemists. ${ }^{[25]}$ The electrochemical reduction reaction has attracted substantial interest in organic synthesis. ${ }^{[26]}$ It avoids the surface activation and post-processing of excessive metal powder. Therefore, it is easy to be used in large-scale industries and significantly reduces the production cost in industrial production. ${ }^{[27]}$ Herein, we report a nickel-promoted electrochemical reductive crosscoupling of trifluoromethyl alkenes and alkyl halides or redox-active esters in an undivided cell (Figure 1d). This reaction enables the synthesis of functionalized and substituted gem-difluoroalkenes under mild electrochemical reduction conditions, therefore obviates the use of stoichiometric amount metal powder or organic reductants. Furthermore, this reaction provides efficient access to biologically interesting molecules containing gem-difluoroethylene moiety.

\section{Results and discussion}

Our investigation began with the synthesis of gemdifluoroalkene 4aa through the nickel-promoted electrochemical reductive cross-coupling of trifluoromethyl alkene 1a with primary alkyl iodide $\mathbf{2 a}$. The reaction parameters were screened systematically, and their impacts on the reaction conversion and coupling efficiency were summarized in Table 1. To our delight, the reaction underwent smoothly and provided $77 \%$ NMR yield and $73 \%$ isolated yield (Entry 1) using $\mathrm{NiBr}_{2}$ (diglyme) as the catalyst, Pybox as the ligand, ${ }^{n} \mathrm{Bu}_{4} \mathrm{NBr}$ as the electrolyte, and dimethyl sulfoxide (DMSO) as the solvent in an undivided cell with $\mathrm{Zn}(+)$ and $\mathrm{Ni}$ foam $(-)$ as electrodes under $5 \mathrm{~mA}$ current for $9 \mathrm{~h}$ at room temperature. $\mathrm{NiCl}_{2}$ or $\mathrm{Ni}(\mathrm{acac})_{2}$ could be used as catalysts, resulting in slightly diminished conversions and yields (Entries 2, 3). Bidentate N-ligands L1 and L2 and another tridentate N-ligand L3 were tested, afford- (a) Applications of gem-difluoroalkenes

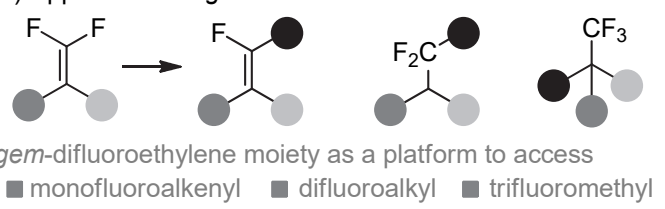

(b) Synthetic routes to gem-difluoroalkenes

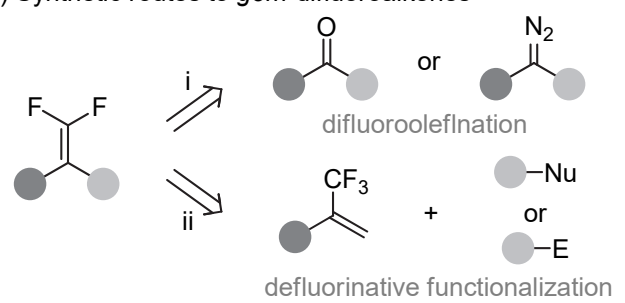

(c) Our previous work: reductive alkylation with zinc

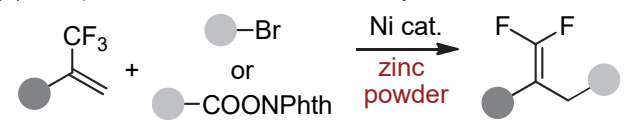

(d) This work: electrochemical reductive cross-coupling

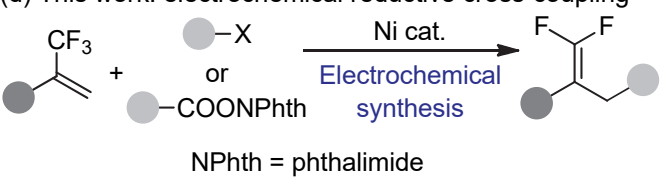

Figure 1 Synthetic routes to access gem-difluoroalkene

ing the desired product in moderate yields (Entries $4 \sim 6$ ). Compared with the data shown in Entries $7 \sim 10$, a satisfactory outcome was obtained when using 1.0 equiv. of ${ }^{n} \mathrm{Bu}_{4} \mathrm{NBr}$ as the suitable electrolyte. $N, N$-Dimethylacetamide (DMAc), $N, N$-dimethylformamide (DMF), and $\mathrm{CH}_{3} \mathrm{CN}$ were frequently-used solvents in electrochemical reactions; when using DMAc and DMF instead of DMSO (Entries 11, 12), the yields decreased to $57 \%$ and $53 \%$, respectively; $\mathrm{CH}_{3} \mathrm{CN}$ was inferior because of the low conversion of alkyl halide (Entry 13). Finally, the excessive amount of alkyl halides ensured the high yield of this reaction (Entries 14, 15).

Control experiments were carried out to clarify the importance of nickel catalyst and electrochemical reduction (Entries 16 22). When the nickel catalyst or electric current was removed, the coupling efficiency of this reaction decreased dramatically (Entries 16 18). When $\mathrm{Fe}(+)$ was used instead of $\mathrm{Zn}(+)$ as the sacrificial anode, a comparable $55 \%$ yield was obtained (Entry 19), while no product was observed when using Fe powder as the reductant (Entry 21). Therefore, these results indirectly explain the electrochemical reduction process rather than the reduction on the metal surface. Ni foam could be replaced with reticulated vitreous carbon (RVC) as cathode, and 73\% NMR yield was obtained (Entry 23). The graphite or platinum plated as cathode provided much lower yields (Entries 25 and 27).

Furthermore, similar outcomes were also obtained when changing the alkyl electrophiles to redox-active esters. The reductive cross-coupling of trifluoromethyl alkenes and redox-active esters under standard conditions gave a grati- 
Table 1 Optimization of reaction conditions ${ }^{a}$
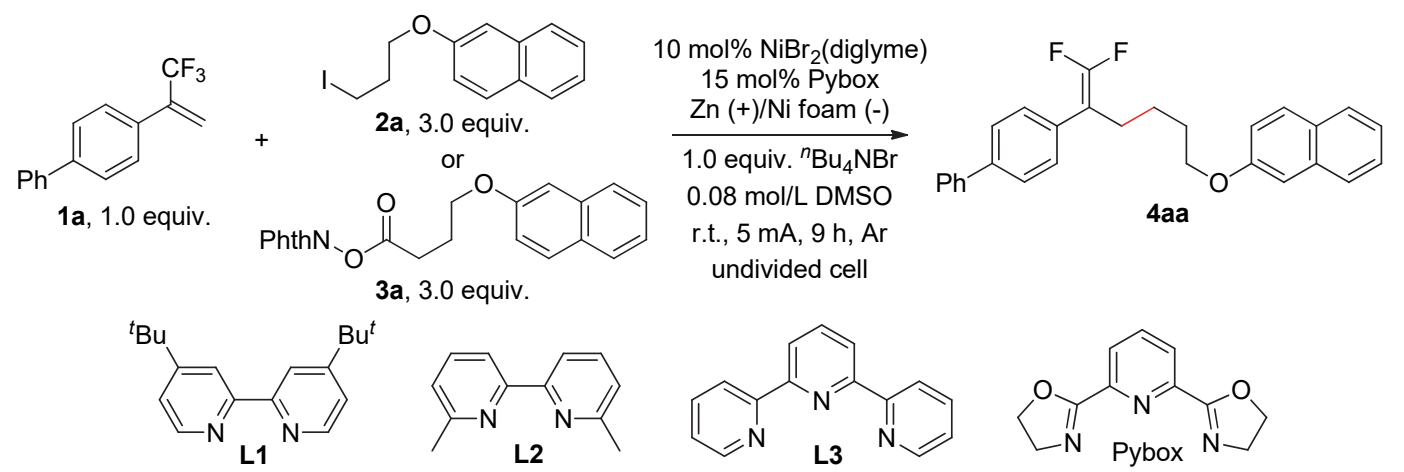

\begin{tabular}{|c|c|c|c|}
\hline Entry & Variation from standard conditions & Conversion $^{b} / \%$ of $\mathbf{1 a}$ & Yield $^{b} / \%$ of $\mathbf{4 a a}$ \\
\hline 1 & No & 81 & $77\left(73^{c}\right)$ \\
\hline 2 & $\mathrm{NiCl}_{2}$ in lieu of $\mathrm{NiBr}_{2}$ (diglyme) & 86 & 76 \\
\hline 3 & $\mathrm{Ni}(\text { acac })_{2}$ in lieu of $\mathrm{NiBr}_{2}$ (diglyme) & 76 & 52 \\
\hline 4 & $\mathbf{L 1}$ in lieu of Pybox & 74 & 40 \\
\hline 5 & $\mathbf{L 2}$ in lieu of Pybox & 62 & 41 \\
\hline 6 & $\mathbf{L 3}$ in lieu of Pybox & 52 & 48 \\
\hline 7 & $\mathrm{KBr}$ in lieu of ${ }^{n} \mathrm{Bu} 4 \mathrm{NBr}$ & 78 & 72 \\
\hline 8 & $\mathrm{LiBr}$ in lieu of ${ }^{n} \mathrm{Bu} 4 \mathrm{NBr}$ & 77 & 62 \\
\hline 9 & 0.5 equiv. ${ }^{n} \mathrm{Bu} 4 \mathrm{NBr}$ & 76 & 75 \\
\hline 10 & 2.0 equiv. ${ }^{n} \mathrm{Bu} 4 \mathrm{NBr}$ & 74 & 70 \\
\hline 11 & DMAc in lieu of DMSO & 66 & 57 \\
\hline 12 & DMF in lieu of DMSO & 78 & 53 \\
\hline 13 & $\mathrm{CH}_{3} \mathrm{CN}$ in lieu of DMSO & 88 & $<2$ \\
\hline 14 & $n(\mathbf{1 a}): n(\mathbf{2 a})=1: 2$ & 84 & 70 \\
\hline 15 & $n(\mathbf{1 a}): n(\mathbf{2 a})=2: 1$ & 66 & 53 \\
\hline 16 & No $\mathrm{NiBr}_{2}$ (diglyme) & 79 & 44 \\
\hline 17 & No electric current & 41 & 39 \\
\hline 18 & No $\mathrm{NiBr}_{2}$ (diglyme), no electric current & 25 & 15 \\
\hline 19 & $\mathrm{Fe}(+)$ in lieu of $\mathrm{Zn}(+)$ & 70 & 55 \\
\hline 20 & $\mathrm{Al}(+)$ in lieu of $\mathrm{Zn}(+)$ & 95 & $<2$ \\
\hline 21 & Fe powder in lieu of electric current & $<2$ & $<2$ \\
\hline 22 & Al powder in lieu of electric current & $<2$ & $<2$ \\
\hline 23 & $\mathrm{RVC}(-)$ in lieu of $\mathrm{Ni}$ foam $(-)$ & 87 & 73 \\
\hline 24 & $\mathrm{RVC}(-)$ in lieu of $\mathrm{Ni}$ foam $(-)$, no $\mathrm{NiBr}_{2}$ (diglyme) & 82 & 10 \\
\hline 25 & Graphite $(-)$ in lieu of Ni foam $(-)$ & 69 & 48 \\
\hline 26 & Graphite $(-)$ in lieu of Ni foam $(-)$, no $\mathrm{NiBr}_{2}$ (diglyme) & 60 & 13 \\
\hline 27 & $\operatorname{Pt}(-)$ in lieu of Ni foam $(-)$ & 81 & 35 \\
\hline 28 & $\mathbf{3 a}$ in lieu of $\mathbf{2 a}$ & 89 & $88\left(84^{c}\right)$ \\
\hline 29 & 3a in lieu of $\mathbf{2 a}$, no $\mathrm{NiBr}_{2}$ (diglyme) & 83 & 64 \\
\hline 30 & 3a in lieu of $\mathbf{2 a}$, no electric current & 70 & 69 \\
\hline 31 & 3a in lieu of $\mathbf{2 a}$, no $\mathrm{NiBr}_{2}$ (diglyme), no electric current & 69 & 67 \\
\hline 32 & $\mathbf{3 a}$ in lieu of $\mathbf{2 a}, \mathrm{Fe}(+)$ in lieu of $\mathrm{Zn}(+)$ & 60 & 42 \\
\hline 33 & 3a in lieu of $\mathbf{2 a}, \mathrm{Al}(+)$ in lieu of $\mathrm{Zn}(+)$ & $>99$ & 31 \\
\hline 34 & 3a in lieu of $\mathbf{2 a}, \mathrm{Fe}$ powder in lieu of electric current & $<2$ & $<2$ \\
\hline 35 & 3a in lieu of $\mathbf{2 a}, \mathrm{Al}$ powder in lieu of electric current & $<2$ & $<2$ \\
\hline 36 & 3a in lieu of $\mathbf{2 a}, n(\mathbf{1 a}): n(\mathbf{3 a})=1: 1.5$ & 50 & 50 \\
\hline 37 & $\mathbf{3 a}$ in lieu of $\mathbf{2 a}, n(\mathbf{1 a}): n(\mathbf{3 a})=1: 2$ & 77 & 52 \\
\hline 38 & 3a in lieu of $\mathbf{2 a}, n(\mathbf{1 a}): n(\mathbf{3 a})=1: 2.5$ & 82 & 76 \\
\hline 39 & 3a in lieu of $\mathbf{2 a}, n(\mathbf{1 a}): n(\mathbf{3 a})=2.5: 1$ & 70 & 37 \\
\hline
\end{tabular}

${ }^{a}$ Reaction was performed on a 0.2 -mmol scale; ${ }^{b}$ Determined by ${ }^{19} \mathrm{~F}$ NMR analysis. Benzotrifluoride was used as an internal standard; ${ }^{c}$ Isolated yield. Diglyme $=$ bis(2-methoxy ethyl)ether..

fying $88 \%$ NMR yield with $84 \%$ isolated yield (Entry 28 ). With the light of contrastive experiments, the nickel cata- lyst and electric current have been proved to be of value for increasing the reaction yield (Entries 29 31). Moreo- 
ver, $\mathrm{Fe}$ or $\mathrm{Al}$ powder can hardly be used in reductive cross-coupling; however, $\mathrm{Fe}(+)$ or $\mathrm{Al}(+)$ can be used in lieu of $\mathrm{Zn}(+)$ as the sacrificial anode (Entries 32 35), indicated the electrochemical reduction process in this reaction. Finally, the addition of nickel catalyst influences the coupling efficiency and may also alter the reaction mechanism. In Xiao's previous work, it has been demonstrated that both radical pathway and organozinc reagent pathway were possible for activating redox-active esters. ${ }^{[28]}$ A radical path was more likely when nickel catalyst was added, and the generation of organozinc reagents was reliable under transition-metal-free conditions. The equivalents of the redox-active esters were tried to be reduced, while 3 equiv. of the redoxactive esters were necessary for obtaining a satisfactory coupling efficiency (Entry 28 vs. Entries 36 39).

The substrate scope of the electrochemical allylic defluorinative reductive alkylation reaction was shown in

Table 2 Scope of electrochemical allylic defluorinative reductive alkylation of trifluoromethyl alkenes ${ }^{a}$

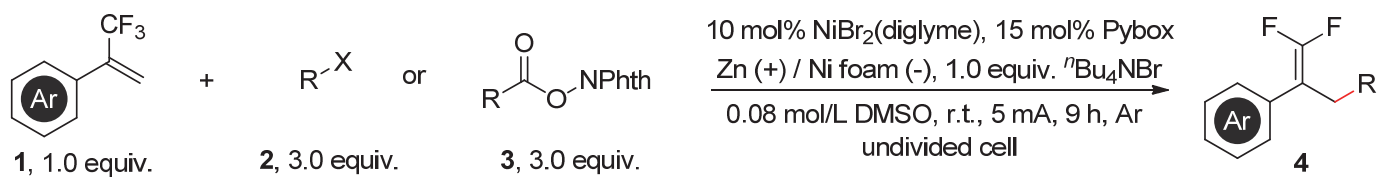

\section{Scope of alkyl halides}<smiles>FC(F)=C(CCCCOc1ccc2ccccc2c1)c1ccc(-c2ccccc2)cc1</smiles>

4aa, $73 \%$ from $1 \mathrm{a} \& 2 \mathrm{a}(X=1)$ $84 \%$ from $1 \mathrm{a} \& 3 a$<smiles>CC(COC(=O)c1ccccc1)CC([Al])=C(F)F</smiles>

4be, $85 \%$ from $1 \mathrm{~b} \& 2 \mathrm{e}(\mathrm{X}=\mathrm{Br})$<smiles>FC(F)=C([Al])CCCCc1ccc(Br)cc1</smiles>

4bi, $52 \%$

from $1 \mathrm{~b} \& 2 \mathrm{i}(X=\mathrm{I})$<smiles>FC(F)=C(CCC(c1ccccc1)c1ccccc1)c1ccc(OCc2ccccc2)cc1</smiles>

4bb, $36 \%$ from $1 \mathrm{~b} \& 2 \mathrm{~b}(X=\mathrm{I})$<smiles>FC(F)=C(CC1CCN([125I])CC1)c1cccc2c1sc1ccccc12</smiles>

4cc, $56 \%$ from $1 c \& 2 c(X=1)$

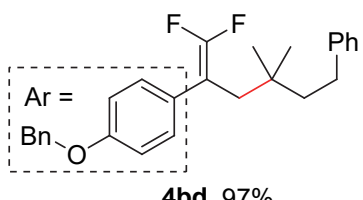

from $1 \mathrm{~b} \& 2 \mathrm{~d}(\mathrm{X}=\mathrm{Br})$

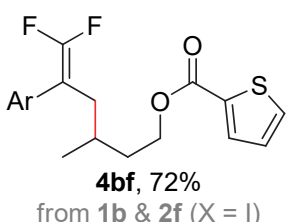<smiles>C=CCCCOc1ccc(CCC(C)(C)CC(Br)=C(F)F)cc1</smiles>

4bg, $90 \%$ from $1 \mathrm{~b} \& 2 \mathrm{~g}(\mathrm{X}=\mathrm{Br})$<smiles>N#Cc1ccc(OCCCCCCCC(Br)=C(F)F)cc1</smiles>

4bk, $56 \%$ from $1 \mathrm{~b} \& 2 \mathrm{k}(\mathrm{X}=\mathrm{I})$<smiles>O=C(OCCCCCCC(=C(F)F)C1CCCCC1)c1ccc(Cc2ccccc2)cc1</smiles>

4bh, $60 \%$ from $1 \mathrm{~b} \& 2 \mathrm{~h}(\mathrm{X}=\mathrm{I})$<smiles>CC(C)(C)OC(=O)Nc1ccc(CCCC([Al])=C(F)F)cc1</smiles>

$4 \mathrm{bl}, 84 \%$ from $1 b$ \& 2 l $(X=\mid)$

\section{Scope of redox-active esters}
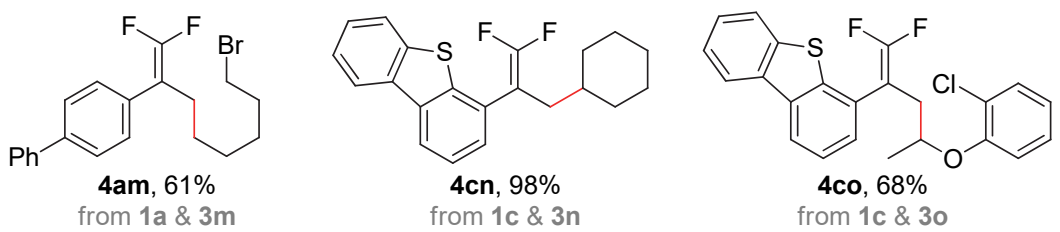<smiles>CC(C)(C)CC(=C(F)F)c1ccc(OCc2ccccc2)cc1</smiles><smiles>O=C([O-])NC(CC([Al])=C(F)F)Cc1ccccc1</smiles>

\section{Scope of trifluoromethyl alkenes}<smiles>CC(C)(C)CC(=C(F)F)c1ccc(C(C)(C)C)cc1</smiles><smiles>O=[Po]([O-])[O-]</smiles><smiles>COc1ccc(C(CBr)=C(F)F)cc1</smiles><smiles>CCCCCCCCCCCCC(=C(F)F)c1ccc(OCc2ccccc2)cc1</smiles><smiles>CC(C)(CCc1ccccc1)CC(=C(F)F)c1ccc(Oc2ccccc2)cc1</smiles><smiles>O=[PH](=O)(O)OCCBr</smiles>
from $1 d \& 2 p(X=B r)$ from $1 e \& 2 p(X=B r)$ from $1 f \& 2 p(X=B r)$ from $1 b \& 2 n(X=I)$<smiles>COc1ccc(C(CCl)=C(F)F)cn1</smiles>

4in, $79 \%$

from 1 i \& $3 n$<smiles>CSc1ccc(C(CC(C)(C)CCc2ccccc2)=C(F)F)cc1</smiles><smiles>CC(C)(CCc1ccccc1)CC(=C(F)F)c1ccccc1N</smiles>

4kd, $34 \%$

from $1 \mathrm{k} \& 2 \mathrm{~d}(\mathrm{X}=\mathrm{Br})$<smiles>C=C(C)C(=O)Nc1cccc(C(CCl)=C(F)F)c1</smiles>

$4 \operatorname{lna} \& 4 \operatorname{lnb}, 81 \%(4 \ln a: 4 \ln b=7: 1)$

from 1 I \& $3 n$

${ }^{a}$ Reaction was performed on a $0.2 \mathrm{mmol}$ scale. Reaction conditions are the same as those for Table 1, Entries 1 and 23 . Isolated yield. ${ }^{b}$ Reaction was performed on $1 \mathrm{mmol}$ scale under $25 \mathrm{~mA}$ current. Isolated yield. $\mathrm{Bn}=$ benzyl, Ts $=$ tosyl, $\mathrm{Bz}=$ benzoyl, Bpin $=$ pinacolato borate, Boc $=$ tert-butoxycarbonyl, $\mathrm{Ac}=$ acetyl, $\mathrm{Cy}=$ cyclohexyl. 
Table 2. A variety of alkyl radical precursors and trifluoromethyl alkenes with different synthetic useful functional groups could be readily transformed to the expected products, and modest to excellent yields (34\% 98\%) were obtained. Both alkyl iodides (e.g., 4aa), alkyl bromides (e.g., 4bd), and alkyl-substituted redox-active esters (e.g., 4cn) were suitable substrates. Not only the primary (e.g., 4aa) and secondary (e.g., 4cc) but also the steric hindrance tertiary (e.g., 4bd, 4bp) alkyl electrophiles posed no problem. Both the cyclic (e.g., 4cc) and the acyclic (e.g., 4be) ones could be used. This electrochemical reduction reaction exhibited good compatibility to many functional groups, such as ether (4bb, $\mathbf{4 f p}, \mathbf{4 b n}$, and $\mathbf{4 g d}$ ), ester (4be), carbamate (4bl, $\mathbf{4 b q})$, and thioether $(\mathbf{4 j d})$. Heterocycles were well tolerated in the reaction, including thiophene (4bf), dibenzothiophene (4cn, 4co), and pyridine (4in). This reaction could be conducted in the presence of basesensitive groups. For instance, alkyl nitrile (4bj), aryl nitrile (4bk), and aryl boronate (4bh) groups posed no problem. More active functional groups, such as unprotected $\mathrm{NH}_{2}$ group (4kd), terminal alkenyl group (4bg), aryl chloride (4co, $4 \mathbf{h p}$ ), and aryl bromide (4bi) were compatible. An interesting example is $\mathbf{4 a m}$; alkyl bromide was proven to be less reactive than alkyl-substituted redox-active ester as the alkyl radical precursor. This chemoselectivity provided opportunities for further manipulations at the surviving alkyl carbon-halogen bonds. In another competition experiment, the standard reaction conditions were applied to a trifluoromethyl alkene substrate containing an acrylamide group (11). The mono-alkylation (4Ina, defluorinative alkylation) and di-alkylation (4lnb, defluorinative alkylation, and Giese addition) products were obtained. And the trifluoromethyl alkene site exhibited a much higher reactivity over the acrylamide site. The reaction of aliphatic substituted trifluoromethyl alkene $(E)-((6,6,6$-trifluorohex4-en-1-yl)oxy)benzene with alkyl bromide $\mathbf{2 p}$ was also tested under standard conditions. Unfortunately, the desired product was not observed; the aliphatic substituted trifluoromethyl alkene $(E)-((6,6,6$-trifluorohex-4-en-1-yl)oxy)benzene was recovered in $90 \%$ NMR yield. Finally, it should be pointed out that there were differences between the alkyl halides and alkyl-substituted redox-active esters when considering the laboratory synthesis methods and reagent sources; the two complement each other. In particular, many $\alpha$-heteroatom alkyl halides may be very difficult and even chemically infeasible to prepare. But the corresponding alkyl-substituted redox-active esters (4co and 4 bq) were accomplished with extreme ease.

This reaction has been used as an efficient tool for the late-stage modification of natural products and drug molecules. As shown in Table 3, the derivatives of fructose (4br), amino acid (4bs), and dehydrocholic acid (4bt) performed well and delivered the desired products smoothly. With these efficient conversions as irresistible proof, it was assured that this electrochemical allylic defluorinative reductive alkylation reaction exhibited good compatibility to diverse functional groups and showed attraction in modifi- cation of physiological activity molecules.

Table 3 Modification of biologically interesting molecules ${ }^{a}$
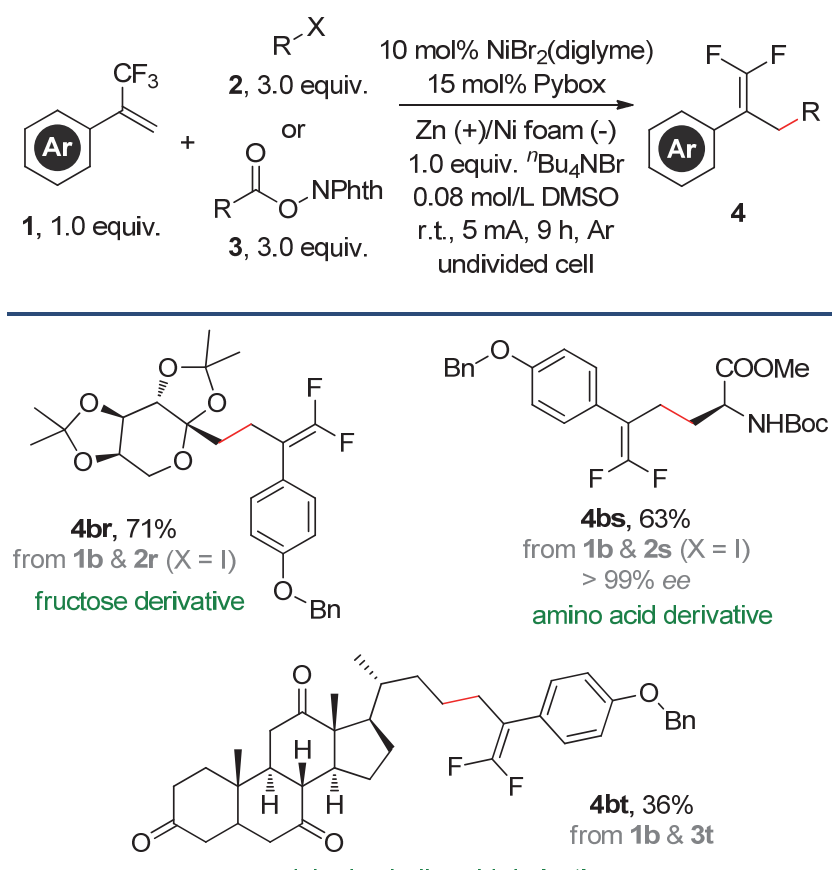

dehydrocholic acid derivative

${ }^{a}$ Reaction was performed on a $0.2 \mathrm{mmol}$ scale. Reaction conditions are the same as those for Table 1, Entries 1 and 23. Isolated yield.

The possible mechanism of this electrochemical allylic defluorinative reductive alkylation reaction was studied (Scheme 1). (Bromomethyl)cyclopropane (2u) was used to participate in the coupling reaction, and only the ringopening product $\mathbf{4 b u}$ was obtained. For the substrate $\mathbf{2 v}$ containing an intramolecular alkenyl group, under the standard conditions, a mixture of cyclized product $4 \mathbf{b v a}$ and linear acyclic product $\mathbf{4 b v b}$ was received. The cyclized product 4 bva may be obtained through the radical cyclization process. Finally, by adding radical inhibitor 2,2,6,6tetramethylpiperidinooxy (TEMPO) to the standard conditions, this reaction has been significantly inhibited in the presence of 0.5 equiv. of TEMPO. And R-TEMPO was isolated. Therefore, this reaction might undergo a radical pathway for activating alkyl electrophiles and was supported by the available evidence.

A proposed reaction mechanism was depicted for this electrochemical reductive coupling reaction (Figure 2). The $\mathrm{NiBr}_{2}$ (diglyme) catalyst was reduced to $\mathrm{Ni}(0)$ active catalyst upon cathodic reduction at the reaction initiation step. Single-electron transfer (SET) from the Ni(0) catalyst to alkyl halides or redox-active esters led to the generation of $\mathrm{Ni}(\mathrm{I})$ species and alkyl radicals. Next, the radical addition to trifluoromethyl alkenes afforded the $\alpha$-trifluoromethyl benzylic radicals, which could be captured by $\mathrm{Ni}(\mathrm{I})$ (Pathway I) or reduced by cathodic reduction (Pathway II). The formed alkylnickel species or anions underwent $\beta$-fluoride elimination to deliver the desired products. The regeneration of $\mathrm{Ni}(0)$ active catalyst was realized through cathodic reduction. However, other possible pathways in- 
<smiles>C=C(c1ccc(OCc2ccccc2)cc1)C(F)(F)F</smiles><smiles>C=CCCCC(=C(F)F)c1ccc(OCc2ccccc2)cc1</smiles>

$2 u$

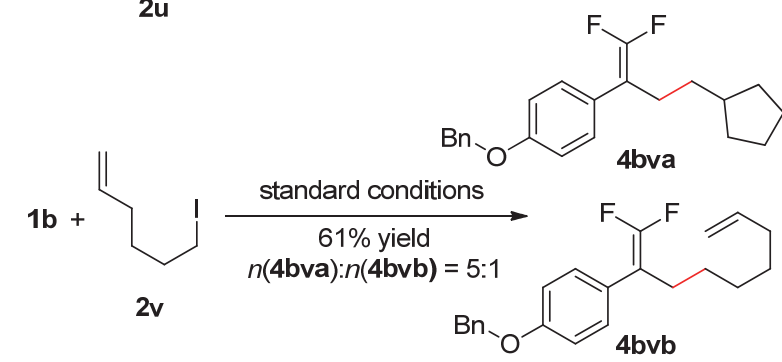

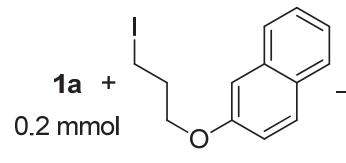

2a, $0.6 \mathrm{mmo}$

\begin{tabular}{clc}
\hline Entry & TEMPO & NMR yield \\
\hline 1 & 0 equiv. & $77 \%$ \\
2 & 0.5 equiv. & $28 \%$ \\
3 & 1.0 equiv. & $19 \%$ \\
4 & 2.0 equiv. & $5 \%$ \\
5 & 3.0 equiv. & $<2 \%$
\end{tabular}

Reaction was performed on a $0.2 \mathrm{mmol}$ scale. Reaction conditions are the same as those for Table 1, Entries 1 and $23 .{ }^{19} \mathrm{~F}$ NMR yield

Scheme 1 Mechanistic probes

volving $\mathrm{Ni}(\mathrm{I}) / \mathrm{Ni}(\mathrm{III})$ catalysis couldn't be ruled out at this stage. ${ }^{[29]}$

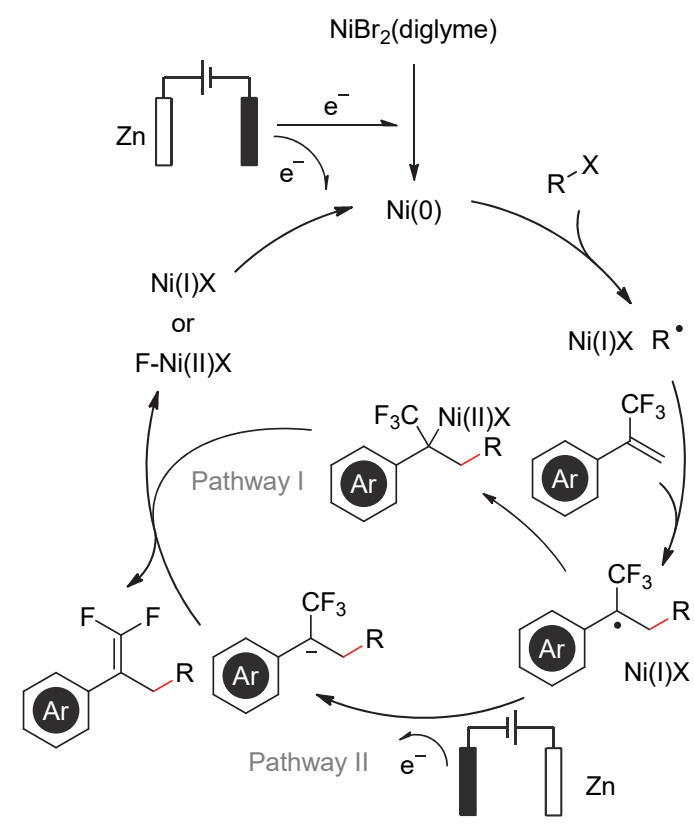

Figure 2 Proposed reaction mechanism

\section{Conclusions}

A nickel-promoted electrochemical reductive crosscoupling reaction was developed to synthesize functionalized and substituted gem-difluoroalkenes. This reaction enables the combination of allylic defluorinative functionalization of trifluoromethyl alkenes, and radical pathway activation of alkyl halides or redox-active esters in an undivided cell obviates the use of stoichiometric amount metal powder or organic reductants. The mild reaction conditions led to high tolerance to many synthetically valuable functional groups and provided efficient access to biologically interesting molecules containing gem-difluoroethylene moiety.

\section{Experimental section}

\subsection{General experimental information}

${ }^{1} \mathrm{H}$ NMR $(500 \mathrm{MHz}),{ }^{13} \mathrm{C}$ NMR $(126 \mathrm{MHz})$, and ${ }^{19} \mathrm{~F}$ NMR (471 MHz) spectra were recorded on a Bruker Avance 500 spectrometer in $\mathrm{CDCl}_{3} .{ }^{19} \mathrm{~F}$ NMR spectra were recorded as ${ }^{19} \mathrm{~F}$ experiment composite pulse decoupling (F19CPD) unless otherwise noted. High-resolution mass spectra (HRMS) were obtained with a Water XEVO G2 Q-Tof (Waters Corporation). Melting points were uncorrected and were recorded on a SG WRS-2A melting point apparatus. Column chromatographic purification of products was accomplished using forced-flow chromatography on Silica Gel (300 400 mesh). Commercially available reagents were purchased from Energy Chemical, J \& K Scientific, Adamas-beta, and Sigma-Aldrich Co., Inc.

\subsection{General procedure for Table 1}

Nickel source (0.02 mmol, $10 \mathrm{~mol} \%)$, ligand (0.03 mmol, $15 \mathrm{~mol} \%)$, and electrolyte $(0.2 \mathrm{mmol}, 1.0$ equiv. $)$ were added to the electrochemical cell equipped with a stir bar. The tube was installed with a sacrificial anode and a $\mathrm{Ni}$ foam as the cathode and was evacuated and filled with argon (three cycles). Then, $1 \mathrm{a}(0.2 \mathrm{mmol}, 1.0$ equiv.) and 2a or 3a ( $0.6 \mathrm{mmol}, 3.0$ equiv.) were added to $2.5 \mathrm{~mL}$ of solvent. Next, the solution was added to the tube and stirred at room temperature $\left(c a .25{ }^{\circ} \mathrm{C}\right)$ for $15 \mathrm{~min}$. The reaction mixture was electrolyzed under a constant current of $5 \mathrm{~mA}$ for $9 \mathrm{~h}$. After the reaction, benzotrifluoride was added to the solution as an internal standard, the yield was determined by ${ }^{19} \mathrm{~F}$ NMR.

\subsection{General procedure for Tables 2 and 3}

$\mathrm{NiBr}_{2}$ (diglyme) (0.02 mmol, $\left.10 \mathrm{~mol} \%\right)$, Pybox (0.03 mmol, $15 \mathrm{~mol} \%$ ), and ${ }^{n} \mathrm{Bu}_{4} \mathrm{NBr}(0.2 \mathrm{mmol}, 1.0$ equiv. $)$ were added to the electrochemical cell equipped with a stir bar. The tube was installed with $\mathrm{Zn}$ as sacrificial anode and $\mathrm{Ni}$ foam as the cathode. Then, it was evacuated and filled with argon (three cycles). Then, 1 ( $0.2 \mathrm{mmol}, 1.0$ equiv.) and 2 or 3 ( $0.6 \mathrm{mmol}, 3.0$ equiv.) were added to $2.5 \mathrm{~mL}$ of DMSO. Next, the solution was added to the tube and stirred at room temperature $\left(c a .25{ }^{\circ} \mathrm{C}\right)$ for $15 \mathrm{~min}$. The reaction mixture was electrolyzed under a constant current of $5 \mathrm{~mA}$ for $9 \mathrm{~h}$. After the reaction, the mixture was puri- 
fied by column chromatography to afford the desired product.

\subsection{Nickel-promoted electrochemical reductive cross-coupling}

2-((5-([1,1'-Biphenyl]-4-yl)-6,6-difluorohex-5-en-1yl)oxy)naphthalene (4aa): Using 4-(3,3,3-trifluoroprop1-en-2-yl)-1,1'-biphenyl (1a) and 2-(3-iodopropoxy)naphthalene (2a), the yield is $73 \%(60.4 \mathrm{mg}$ as colorless oil) after column chromatography; using 4-(3,3,3-trifluoroprop-1-en-2-yl)-1,1'-biphenyl (1a) and 1,3-dioxoisoindolin-2-yl 4-(naphthalen-2-yloxy)butanoate (3a), the yield is $84 \%$ (69.8 $\mathrm{mg}$ as colorless oil) after column chromatography. ${ }^{1} \mathrm{H} \mathrm{NMR}\left(500 \mathrm{MHz}, \mathrm{CDCl}_{3}\right) \delta: 7.89 \sim 7.73(\mathrm{~m}, 3 \mathrm{H})$, $7.73 \sim 7.59(\mathrm{~m}, 4 \mathrm{H}), 7.58 \sim 7.34(\mathrm{~m}, 7 \mathrm{H}), 7.24 \sim 7.12(\mathrm{~m}$, $2 \mathrm{H}), 4.11(\mathrm{t}, J=6.4 \mathrm{~Hz}, 2 \mathrm{H}), 2.62(\mathrm{tt}, J=7.6,2.4 \mathrm{~Hz}, 2 \mathrm{H})$, $1.99 \sim 1.87(\mathrm{~m}, 2 \mathrm{H}), 1.77 \sim 1.63(\mathrm{~m}, 2 \mathrm{H}) ;{ }^{13} \mathrm{C}$ NMR $(126$ $\left.\mathrm{MHz}, \mathrm{CDCl}_{3}\right) \delta: 157.1,153.9(\mathrm{dd}, J=289.8,288.0 \mathrm{~Hz})$, $140.6,140.2$, 134.7, 132.6, 129.5, 129.1, 128.9, 128.7 (dd, $J=3.4,3.4 \mathrm{~Hz}), 127.8,127.5,127.2,127.1,126.8,126.4$, 123.6, 119.1, 106.7, 92.0 (dd, $J=19.3,15.2 \mathrm{~Hz}), 67.5$, 28.6, 27.3, 24.4 (dd, $J=2.5,2.5 \mathrm{~Hz}) ;{ }^{19} \mathrm{~F}$ NMR (471 MHz, $\left.\mathrm{CDCl}_{3}\right) \delta:-90.70(\mathrm{~d}, J=43.6 \mathrm{~Hz}),-90.80(\mathrm{~d}, J=43.3$ $\mathrm{Hz}$ ); ESI-HRMS calcd for $\mathrm{C}_{28} \mathrm{H}_{25} \mathrm{~F}_{2} \mathrm{O}(\mathrm{M}+\mathrm{H})^{+} 415.1868$, found 415.1872 .

(4-(4-(Benzyloxy)phenyl)-5,5-difluoropent-4-ene-1,1diyl)dibenzene (4bb): The yield is $36 \%$ (31.5 mg as colorless oil) after column chromatography. ${ }^{1} \mathrm{H}$ NMR (500 $\left.\mathrm{MHz}, \mathrm{CDCl}_{3}\right) \delta: 7.45 \sim 7.29(\mathrm{~m}, 5 \mathrm{H}), 7.27 \sim 7.20(\mathrm{~m}, 4 \mathrm{H})$, $7.19 \sim 7.12(\mathrm{~m}, 8 \mathrm{H}), 6.98 \sim 6.88(\mathrm{~m}, 2 \mathrm{H}), 5.05(\mathrm{~s}, 2 \mathrm{H})$, $3.87(\mathrm{t}, J=7.8 \mathrm{~Hz}, 1 \mathrm{H}), 2.32(\mathrm{tt}, J=8.1,2.4 \mathrm{~Hz}, 2 \mathrm{H})$, $2.16 \sim 2.02(\mathrm{~m}, 2 \mathrm{H}) ;{ }^{13} \mathrm{C} \mathrm{NMR}\left(126 \mathrm{MHz}, \mathrm{CDCl}_{3}\right) \delta$ : $158.0,153.5$ (dd, $J=289.5,286.5 \mathrm{~Hz}), 144.6,137.0,129.5$ (dd, $J=3.3,3.3 \mathrm{~Hz}$ ), 128.8, 128.6, 128.2, 127.9, 127.6, $126.4,126.0$ (dd, $J=3.2,3.2 \mathrm{~Hz}), 115.0,91.7$ (dd, $J=21.7$, $13.3 \mathrm{~Hz}), 70.2,51.0,33.9(\mathrm{dd}, J=2.1,2.1 \mathrm{~Hz}), 26.4 ;{ }^{19} \mathrm{~F}$ NMR (471 MHz, $\left.\mathrm{CDCl}_{3}\right) \delta:-91.96(\mathrm{~d}, J=45.2 \mathrm{~Hz})$, $-92.27(\mathrm{~d}, J=45.5 \mathrm{~Hz})$; ESI-HRMS calcd for $\mathrm{C}_{30} \mathrm{H}_{27} \mathrm{~F}_{2} \mathrm{O}$ $(\mathrm{M}+\mathrm{H})^{+}$441.2024, found 441.2043.

4-(2-(Dibenzo[b,d]thiophen-4-yl)-3,3-difluoroallyl)-1tosylpiperidine (4cc): The yield is $56 \%(56.2 \mathrm{mg}$ as colorless oil) after column chromatography. ${ }^{1} \mathrm{H}$ NMR (500 $\left.\mathrm{MHz}, \mathrm{CDCl}_{3}\right) \delta: 8.16 \sim 8.05(\mathrm{~m}, 2 \mathrm{H}), 7.87 \sim 7.82(\mathrm{~m}, 1 \mathrm{H})$, $7.60 \sim 7.54(\mathrm{~m}, 2 \mathrm{H}), 7.48 \sim 7.42(\mathrm{~m}, 3 \mathrm{H}), 7.28 \sim 7.20(\mathrm{~m}$, $3 \mathrm{H}), 3.77 \sim 3.67(\mathrm{~m}, 2 \mathrm{H}), 2.48 \sim 2.40(\mathrm{~m}, 2 \mathrm{H}), 2.36(\mathrm{~s}$, $3 \mathrm{H}), 2.06(\mathrm{td}, J=12.0,2.7 \mathrm{~Hz}, 2 \mathrm{H}), 1.81 \sim 1.74(\mathrm{~m}, 2 \mathrm{H})$, $1.42 \sim 1.31(\mathrm{~m}, 2 \mathrm{H}), 1.16 \sim 1.05(\mathrm{~m}, 1 \mathrm{H}) ;{ }^{13} \mathrm{C}$ NMR $(126$ $\left.\mathrm{MHz}, \mathrm{CDCl}_{3}\right) \delta: 153.7$ (dd, $\left.J=290.8,289.8 \mathrm{~Hz}\right), 143.4$, 139.7 (dd, $J=2.0,2.0 \mathrm{~Hz}), 139.1,136.2,135.7,133.2$, 129.7, $128.6(\mathrm{dd}, J=1.3,1.3 \mathrm{~Hz}), 127.8,127.4(\mathrm{dd}, J=1.3$, $1.3 \mathrm{~Hz}), 127.2,124.9,124.7,122.8,121.9,121.2,89.6$ (dd, $J=23.4,16.1 \mathrm{~Hz}), 46.3,34.8,33.7(\mathrm{dd}, J=2.5,2.5 \mathrm{~Hz})$, $31.4,21.6 ;{ }^{19} \mathrm{~F}$ NMR $\left(471 \mathrm{MHz}, \mathrm{CDCl}_{3}\right) \delta$ : $-85.37(\mathrm{~d}$, $J=36.4 \mathrm{~Hz}),-90.40(\mathrm{~d}, J=37.3 \mathrm{~Hz})$; ESI-HRMS calcd for $\mathrm{C}_{27} \mathrm{H}_{26} \mathrm{~F}_{2} \mathrm{NO}_{2} \mathrm{~S}_{2}(\mathrm{M}+\mathrm{H})^{+}$498.1368, found 498.1373 . The NMR data were consistent with those reported in previous literature. ${ }^{[23 c]}$
1-(Benzyloxy)-4-(1,1-difluoro-4,4-dimethyl-6-phenylhex-1-en-2-yl)benzene (4bd): The yield is $97 \%(78.5 \mathrm{mg}$ as colorless oil) after column chromatography. ${ }^{1} \mathrm{H}$ NMR $\left(400 \mathrm{MHz}, \mathrm{CDCl}_{3}\right) \delta: 7.48 \sim 7.30(\mathrm{~m}, 5 \mathrm{H}), 7.26 \sim 7.18(\mathrm{~m}$, $4 \mathrm{H}), 7.17 \sim 7.10(\mathrm{~m}, 1 \mathrm{H}), 7.05 \sim 6.89(\mathrm{~m}, 4 \mathrm{H}), 5.05(\mathrm{~s}$, $2 \mathrm{H}), 2.53 \sim 2.43(\mathrm{~m}, 2 \mathrm{H}), 2.38(\mathrm{t}, J=2.4 \mathrm{~Hz}, 2 \mathrm{H}), 1.50 \sim$ $1.36(\mathrm{~m}, 2 \mathrm{H}), 0.86(\mathrm{~s}, 6 \mathrm{H}) ;{ }^{13} \mathrm{C} \mathrm{NMR}\left(101 \mathrm{MHz}, \mathrm{CDCl}_{3}\right) \delta$ : $158.0,154.5$ (dd, $J=287.4,286.5 \mathrm{~Hz}), 143.2,137.0,129.8$ (dd, $J=2.8,2.8 \mathrm{~Hz}), 128.7,128.4,128.4,128.2,128.1$ (dd, $J=4.8,2.6 \mathrm{~Hz}), 127.6,125.7,114.9,90.3$ (dd, $J=21.3$, $13.6 \mathrm{~Hz}), 70.2$, 44.5, 39.2, 35.5 (dd, $J=2.6,2.6 \mathrm{~Hz}), 30.8$, 27.6; ${ }^{19} \mathrm{~F} \mathrm{NMR}\left(376 \mathrm{MHz}, \mathrm{CDCl}_{3}\right) \delta:-90.17$ (d, $J=42.5$ $\mathrm{Hz}),-92.56(\mathrm{~d}, J=42.9 \mathrm{~Hz})$; ESI-HRMS calcd for $\mathrm{C}_{27} \mathrm{H}_{28} \mathrm{~F}_{2} \mathrm{ONa}(\mathrm{M}+\mathrm{Na})^{+}$429.2000, found 429.2012.

4-(4-(Benzyloxy)phenyl)-5,5-difluoro-2-methylpent-4en-1-yl benzoate (4be): The yield is $85 \%(71.7 \mathrm{mg}$ as colorless oil) after column chromatography. ${ }^{1} \mathrm{H}$ NMR $(500$ $\left.\mathrm{MHz}, \mathrm{CDCl}_{3}\right) \delta: 8.10 \sim 7.94(\mathrm{~m}, 2 \mathrm{H}), 7.59 \sim 7.51(\mathrm{~m}, 1 \mathrm{H})$, $7.47 \sim 7.35(\mathrm{~m}, 6 \mathrm{H}), 7.35 \sim 7.29(\mathrm{~m}, 1 \mathrm{H}), 7.28 \sim 7.22(\mathrm{~m}$, $2 \mathrm{H}), 6.99 \sim 6.91(\mathrm{~m}, 2 \mathrm{H}), 5.04(\mathrm{~s}, 2 \mathrm{H}), 4.25 \sim 4.06(\mathrm{~m}$, $2 \mathrm{H}), 2.63 \sim 2.30(\mathrm{~m}, 2 \mathrm{H}), 2.10 \sim 1.86(\mathrm{~m}, 1 \mathrm{H}), 1.01(\mathrm{~d}, J=$ $6.8 \mathrm{~Hz}, 3 \mathrm{H}) ;{ }^{13} \mathrm{C}$ NMR $\left(126 \mathrm{MHz}, \mathrm{CDCl}_{3}\right) \delta: 166.6,158.1$, 154.1 (dd, $J=288.1,288.1 \mathrm{~Hz}), 137.0,133.0,130.4,129.6$, $129.5(\mathrm{dd}, J=3.2,3.2 \mathrm{~Hz}), 128.7,128.5,128.1,127.6$, 125.8, 115.0, $90.2(\mathrm{dd}, J=17.8,17.8 \mathrm{~Hz}), 70.1,68.9,31.6$, $31.3(\mathrm{dd}, J=2.5,2.5 \mathrm{~Hz}), 16.7$; ${ }^{19} \mathrm{~F}$ NMR (471 MHz, $\left.\mathrm{CDCl}_{3}\right) \delta$ : -91.48 ; ESI-HRMS calcd for $\mathrm{C}_{26} \mathrm{H}_{24} \mathrm{~F}_{2} \mathrm{O}_{3} \mathrm{Na}$ $(\mathrm{M}+\mathrm{Na})^{+}$445.1586, found 445.1591.

5-(4-(Benzyloxy)phenyl)-6,6-difluoro-3-methylhex-5en-1-yl thiophene-2-carboxylate (4bf): The yield is $72 \%$ (63.9 $\mathrm{mg}$ as colorless oil) after column chromatography. ${ }^{1} \mathrm{H}$ NMR $\left(500 \mathrm{MHz}, \mathrm{CDCl}_{3}\right) \delta: 7.75 \sim 7.65(\mathrm{~m}, 1 \mathrm{H}), 7.56 \sim$ $7.48(\mathrm{~m}, 1 \mathrm{H}), 7.47 \sim 7.29(\mathrm{~m}, 5 \mathrm{H}), 7.25 \sim 7.18(\mathrm{~m}, 2 \mathrm{H})$, $7.10 \sim 7.03(\mathrm{~m}, 1 \mathrm{H}), 6.96 \sim 6.87(\mathrm{~m}, 2 \mathrm{H}), 5.04(\mathrm{~s}, 2 \mathrm{H})$, $4.39 \sim 4.21(\mathrm{~m}, 2 \mathrm{H}), 2.48 \sim 2.23(\mathrm{~m}, 1 \mathrm{H}), 1.86 \sim 1.77(\mathrm{~m}$, $1 \mathrm{H}), 1.72 \sim 1.63(\mathrm{~m}, 1 \mathrm{H}), 1.61 \sim 1.52(\mathrm{~m}, 1 \mathrm{H}), 0.95(\mathrm{~d}, J=$ $6.7 \mathrm{~Hz}, 3 \mathrm{H}) ;{ }^{13} \mathrm{C} \mathrm{NMR}\left(126 \mathrm{MHz}, \mathrm{CDCl}_{3}\right) \delta: 162.3,158.1$, 154.1 (dd, $J=287.7,287.7 \mathrm{~Hz}), 137.0,134.1,133.4,132.4$, $129.5(\mathrm{dd}, J=3.2,3.2 \mathrm{~Hz}), 128.7,128.2,127.8,127.6$, 126.0, 114.9, 90.7 (dd, $J=17.5,17.5 \mathrm{~Hz}), 70.1,63.3,35.1$, 35.0, $28.2(\mathrm{dd}, J=2.5,2.5 \mathrm{~Hz}), 19.3 ;{ }^{19} \mathrm{~F}$ NMR (471 MHz, $\left.\mathrm{CDCl}_{3}\right) \delta$ : -92.03 ; ESI-HRMS calcd for $\mathrm{C}_{25} \mathrm{H}_{24} \mathrm{~F}_{2} \mathrm{O}_{3} \mathrm{SNa}$ $(\mathrm{M}+\mathrm{Na})^{+}$465.1306, found 465.1307.

1-(Benzyloxy)-4-(1,1-difluoro-4,4-dimethyl-6-(4-(pent4-en-1-yloxy)phenyl)hex-1-en-2-yl)benzene (4bg): The yield is $90 \%$ (88.1 $\mathrm{mg}$ as colorless oil) after column chromatography. ${ }^{1} \mathrm{H}$ NMR $\left(500 \mathrm{MHz}, \mathrm{CDCl}_{3}\right) \delta: 7.50 \sim 7.35$ $(\mathrm{m}, 5 \mathrm{H}), 7.30 \sim 7.25(\mathrm{~m}, 2 \mathrm{H}), 7.01 \sim 6.96(\mathrm{~m}, 2 \mathrm{H}), 6.93 \sim$ $6.87(\mathrm{~m}, 2 \mathrm{H}), 6.83 \sim 6.77(\mathrm{~m}, 2 \mathrm{H}), 5.95 \sim 5.82(\mathrm{~m}, 1 \mathrm{H})$, $5.13 \sim 4.99(\mathrm{~m}, 4 \mathrm{H}), 3.95(\mathrm{t}, J=6.5 \mathrm{~Hz}, 2 \mathrm{H}), 2.48 \sim 2.36$ $(\mathrm{m}, 4 \mathrm{H}), 2.30 \sim 2.22(\mathrm{~m}, 2 \mathrm{H}), 1.93 \sim 1.84(\mathrm{~m}, 2 \mathrm{H}), 1.47 \sim$ $1.40(\mathrm{~m}, 2 \mathrm{H}), 0.88(\mathrm{~s}, 6 \mathrm{H}) ;{ }^{13} \mathrm{C} \mathrm{NMR}\left(126 \mathrm{MHz}, \mathrm{CDCl}_{3}\right) \delta$ : 158.0, 157.2, 154.5 (dd, $J=287.8,286.4 \mathrm{~Hz}), 138.0,137.0$, 135.1, $129.8(\mathrm{dd}, J=2.8,2.8 \mathrm{~Hz}), 129.5(\mathrm{dd}, J=3.2,3.2$ Hz), 129.2, 128.7, 128.1, 127.6, 115.2, 114.9, 114.4, 90.4 (dd, $J=21.4,13.6 \mathrm{~Hz}), 70.2,67.3,44.8,39.2,35.4$ (dd, $J=$ 2.5, $2.5 \mathrm{~Hz}), 30.3,29.8,28.6,27.6 ;{ }^{19} \mathrm{~F}$ NMR (471 MHz, 
$\left.\mathrm{CDCl}_{3}\right) \delta:-90.22(\mathrm{~d}, J=43.4 \mathrm{~Hz}),-92.60(\mathrm{~d}, J=43.3$ $\mathrm{Hz})$; ESI-HRMS calcd for $\mathrm{C}_{32} \mathrm{H}_{37} \mathrm{~F}_{2} \mathrm{O}_{2}(\mathrm{M}+\mathrm{H}){ }^{+}$ 491.2756, found 491.2757.

8-(4-(Benzyloxy)phenyl)-9,9-difluoronon-8-en-1-yl 4(4,4,5,5-tetramethyl-1,3,2-dioxaborolan-2-yl)benzoate (4bh): The yield is $60 \%$ ( $70.7 \mathrm{mg}$ as colorless oil) after column chromatography. ${ }^{1} \mathrm{H}$ NMR $\left(400 \mathrm{MHz}, \mathrm{CDCl}_{3}\right) \delta$ : $8.07 \sim 7.95(\mathrm{~m}, 2 \mathrm{H}), 7.92 \sim 7.80(\mathrm{~m}, 2 \mathrm{H}), 7.50 \sim 7.30(\mathrm{~m}$, $5 \mathrm{H}), 7.25 \sim 7.17(\mathrm{~m}, 2 \mathrm{H}), 7.03 \sim 6.89(\mathrm{~m}, 2 \mathrm{H}), 5.06(\mathrm{~s}$, $2 \mathrm{H}), 4.30(\mathrm{t}, J=6.7 \mathrm{~Hz}, 2 \mathrm{H}), 2.45 \sim 2.27(\mathrm{~m}, 2 \mathrm{H}), 1.80 \sim$ $1.69(\mathrm{~m}, 2 \mathrm{H}), 1.42 \sim 1.29(\mathrm{~m}, 20 \mathrm{H}) ;{ }^{13} \mathrm{C} \mathrm{NMR}(101 \mathrm{MHz}$, $\left.\mathrm{CDCl}_{3}\right) \delta: 166.8,158.0,153.6(\mathrm{dd}, J=287.4,287.4 \mathrm{~Hz})$, 137.0, 134.8, 132.8, 129.5 (dd, $J=3.3,3.3 \mathrm{~Hz}), 128.7$, $128.7,128.1,127.6,126.3,114.9,91.9(\mathrm{dd}, J=17.4,17.4$ Hz), 84.3, 70.1, 65.3, 29.1, 29.0, 28.7, 27.7, 27.7, 26.1, $25.0 ;{ }^{19} \mathrm{~F}$ NMR $\left(376 \mathrm{MHz}, \mathrm{CDCl}_{3}\right) \delta:-92.75 ;{ }^{11} \mathrm{~B}$ NMR $\left(160 \mathrm{MHz}, \mathrm{CDCl}_{3}\right) \delta$ : 30.46; ESI-HRMS calcd for $\mathrm{C}_{35} \mathrm{H}_{42} \mathrm{BF}_{2} \mathrm{O}_{5}(\mathrm{M}+\mathrm{H})^{+}$591.3088, found 591.3098.

1-(Benzyloxy)-4-(6-(4-bromophenyl)-1,1-difluorohex-1en-2-yl)benzene (4bi): The yield is $52 \%(47.7 \mathrm{mg}$ as colorless oil) after column chromatography. ${ }^{1} \mathrm{H}$ NMR (500 $\left.\mathrm{MHz}, \mathrm{CDCl}_{3}\right) \delta: 7.49 \sim 7.31(\mathrm{~m}, 7 \mathrm{H}), 7.23 \sim 7.18(\mathrm{~m}, 2 \mathrm{H})$, $7.03 \sim 6.92(\mathrm{~m}, 4 \mathrm{H}), 5.08(\mathrm{~s}, 2 \mathrm{H}), 2.58 \sim 2.48(\mathrm{~m}, 2 \mathrm{H})$, $2.45 \sim 2.32(\mathrm{~m}, 2 \mathrm{H}), 1.66 \sim 1.53(\mathrm{~m}, 2 \mathrm{H}), 1.45 \sim 1.33(\mathrm{~m}$, $2 \mathrm{H}) ;{ }^{13} \mathrm{C} \mathrm{NMR}\left(126 \mathrm{MHz}, \mathrm{CDCl}_{3}\right) \delta: 158.0,153.6(\mathrm{dd}, J=$ 286.4, 285.9 Hz), 141.4, 137.0, 131.4, 130.3, 129.5 (dd, $J=3.3,3.3 \mathrm{~Hz}), 128.8,128.2,127.6,126.1(\mathrm{dd}, J=2.1,2.1$ $\mathrm{Hz}), 119.5,114.9,91.7(\mathrm{dd}, J=20.9,13.7 \mathrm{~Hz}), 70.2,35.0$, 30.6, 27.5, 27.1; ${ }^{19} \mathrm{~F}$ NMR (471 MHz, $\left.\mathrm{CDCl}_{3}\right) \delta$ : -92.42 (d, $J=46.6 \mathrm{~Hz}),-92.56(\mathrm{~d}, J=46.4 \mathrm{~Hz})$; ESI-HRMS calcd for $\mathrm{C}_{25} \mathrm{H}_{23} \mathrm{BrF}_{2} \mathrm{ONa}(\mathrm{M}+\mathrm{Na})^{+}$479.0793, found 479.0791.

7-(4-(Benzyloxy)phenyl)-8,8-difluorooct-7-enenitrile (4bj): The yield is $45 \%$ (30.5 $\mathrm{mg}$ as colorless oil) after column chromatography. ${ }^{1} \mathrm{H}$ NMR $\left(500 \mathrm{MHz}, \mathrm{CDCl}_{3}\right) \delta$ : $7.47 \sim 7.32(\mathrm{~m}, 5 \mathrm{H}), 7.24 \sim 7.19(\mathrm{~m}, 2 \mathrm{H}), 7.01 \sim 6.94(\mathrm{~m}$, 2H), $5.08(\mathrm{~s}, 2 \mathrm{H}), 2.39(\mathrm{tt}, J=7.2,2.4 \mathrm{~Hz}, 2 \mathrm{H}), 2.29(\mathrm{t}, J=$ $7.1 \mathrm{~Hz}, 2 \mathrm{H}), 1.67 \sim 1.58(\mathrm{~m}, 2 \mathrm{H}), 1.49 \sim 1.35(\mathrm{~m}, 4 \mathrm{H}) ;{ }^{13} \mathrm{C}$ NMR (126 MHz, $\left.\mathrm{CDCl}_{3}\right) \delta: 158.1,153.6$ (dd, $J=289.3$, $286.2 \mathrm{~Hz}$ ), 137.0, 129.5 (dd, $J=3.3,3.3 \mathrm{~Hz}$ ), 128.7, 128.2, 127.6, 125.9 (dd, $J=2.1,2.1 \mathrm{~Hz}), 119.7,115.0,91.5$ (dd, $J=20.9,14.3 \mathrm{~Hz}$ ), 70.2, 28.0, 27.4, 26.9 (dd, $J=2.5,2.5$ $\mathrm{Hz}), 25.2,17.2 ;{ }^{19} \mathrm{~F}$ NMR $\left(471 \mathrm{MHz}, \mathrm{CDCl}_{3}\right) \delta$ : -92.26 (d, $J=45.9 \mathrm{~Hz}),-92.42(\mathrm{~d}, J=46.0 \mathrm{~Hz})$; ESI-HRMS calcd for $\mathrm{C}_{21} \mathrm{H}_{21} \mathrm{~F}_{2} \mathrm{NONa}(\mathrm{M}+\mathrm{Na})^{+}$364.1483, found 364.1485 .

4-((8-(4-(Benzyloxy)phenyl)-9,9-difluoronon-8-en-1yl)oxy)benzonitrile (4bk): The yield is $56 \%(51.6 \mathrm{mg}$ as colorless oil) after column chromatography. ${ }^{1} \mathrm{H}$ NMR (500 $\left.\mathrm{MHz}, \mathrm{CDCl}_{3}\right) \delta: 7.61 \sim 7.53(\mathrm{~m}, 2 \mathrm{H}), 7.48 \sim 7.29(\mathrm{~m}, 5 \mathrm{H})$, $7.25 \sim 7.19(\mathrm{~m}, 2 \mathrm{H}), 7.00 \sim 6.88(\mathrm{~m}, 4 \mathrm{H}), 5.07(\mathrm{~s}, 2 \mathrm{H})$, $3.97(\mathrm{t}, J=6.5 \mathrm{~Hz}, 2 \mathrm{H}), 2.45 \sim 2.26(\mathrm{~m}, 2 \mathrm{H}), 1.82 \sim 1.73$ $(\mathrm{m}, 2 \mathrm{H}), 1.45 \sim 1.31(\mathrm{~m}, 8 \mathrm{H}) ;{ }^{13} \mathrm{C} \mathrm{NMR}(126 \mathrm{MHz}$, $\left.\mathrm{CDCl}_{3}\right) \delta: 162.5,158.0,153.6(\mathrm{dd}, J=288.3,286.5 \mathrm{~Hz})$, $137.0,134.0,129.5$ (dd, $J=3.0,3.0 \mathrm{~Hz}), 128.7,128.1$, 127.6, $126.3(\mathrm{dd}, J=2.4,2.4 \mathrm{~Hz}), 119.4,115.3,114.9$, 103.7, 91.9 (dd, $J=19.7,14.9 \mathrm{~Hz}$ ), 70.1, 68.4, 29.0, 29.0,
28.9, 27.7, $27.7(\mathrm{dd}, J=2.2,2.2 \mathrm{~Hz}), 25.9 ;{ }^{19} \mathrm{~F} \mathrm{NMR}(471$ $\left.\mathrm{MHz}, \mathrm{CDCl}_{3}\right) \delta:-92.63(\mathrm{~d}, J=46.9 \mathrm{~Hz}),-92.74(\mathrm{~d}$, $J=46.8 \mathrm{~Hz}$ ); ESI-HRMS calcd for $\mathrm{C}_{29} \mathrm{H}_{29} \mathrm{~F}_{2} \mathrm{NO}_{2} \mathrm{Na}(\mathrm{M}+$ $\mathrm{Na})^{+}$484.2059, found 484.2064.

tert-Butyl (4-(4-(4-(benzyloxy)phenyl)-5,5-difluoropent4-en-1-yl)phenyl) carbamate (4bl): The yield is $84 \%(80.1$ $\mathrm{mg}$ as colorless oil) after column chromatography. ${ }^{1} \mathrm{H}$ NMR $\left(500 \mathrm{MHz}, \mathrm{CDCl}_{3}\right) \delta: 7.47 \sim 7.32(\mathrm{~m}, 5 \mathrm{H}), 7.26 \sim$ $7.18(\mathrm{~m}, 4 \mathrm{H}), 7.07 \sim 7.01(\mathrm{~m}, 2 \mathrm{H}), 7.00 \sim 6.93(\mathrm{~m}, 2 \mathrm{H})$, $6.48(\mathrm{~s}, 1 \mathrm{H}), 5.07(\mathrm{~s}, 2 \mathrm{H}), 2.56(\mathrm{t}, J=7.7 \mathrm{~Hz}, 2 \mathrm{H}), 2.47 \sim$ $2.33(\mathrm{~m}, 2 \mathrm{H}), 1.70 \sim 1.62(\mathrm{~m}, 2 \mathrm{H}), 1.53(\mathrm{~s}, 9 \mathrm{H}) ;{ }^{13} \mathrm{C} \mathrm{NMR}$ $\left(126 \mathrm{MHz}, \mathrm{CDCl}_{3}\right) \delta: 158.0,153.4(\mathrm{dd}, J=287.2,216.7$ $\mathrm{Hz}), 137.0,136.7,136.3,129.5$ (dd, $J=3.2,3.2 \mathrm{~Hz}), 128.9$, $128.7,128.1,127.6,126.1,118.8,114.9,91.7$ (dd, $J=16.8$, $16.8 \mathrm{~Hz}), 80.4,70.1,34.6,29.5$ (dd, $J=2.7,2.7 \mathrm{~Hz}), 28.5$, 27.3; ${ }^{19} \mathrm{~F}$ NMR $\left(471 \mathrm{MHz}, \mathrm{CDCl}_{3}\right) \delta$ : - 92.34; ESIHRMS calcd for $\mathrm{C}_{29} \mathrm{H}_{31} \mathrm{~F}_{2} \mathrm{NO}_{3} \mathrm{Na}(\mathrm{M}+\mathrm{Na})^{+}$502.2164, found 502.2165 .

4-(8-Bromo-1,1-difluorooct-1-en-2-yl)-1,1'-biphenyl (4am): The yield is $61 \%$ (46.1 mg as colorless oil) after column chromatography. ${ }^{1} \mathrm{H}$ NMR $\left(500 \mathrm{MHz}, \mathrm{CDCl}_{3}\right) \delta$ : $7.61(\mathrm{td}, J=7.3,6.6,1.8 \mathrm{~Hz}, 4 \mathrm{H}), 7.46(\mathrm{t}, J=7.6 \mathrm{~Hz}, 2 \mathrm{H})$, $7.42 \sim 7.33(\mathrm{~m}, 3 \mathrm{H}), 3.39(\mathrm{t}, J=6.8 \mathrm{~Hz}, 2 \mathrm{H}), 2.45(\mathrm{tt}, J=$ $7.8,2.4 \mathrm{~Hz}, 2 \mathrm{H}), 1.89 \sim 1.78(\mathrm{~m}, 2 \mathrm{H}), 1.48 \sim 1.30(\mathrm{~m}, 6 \mathrm{H})$; ${ }^{13} \mathrm{C} \mathrm{NMR}\left(126 \mathrm{MHz}, \mathrm{CDCl}_{3}\right) \delta: 153.8(\mathrm{dd}, J=288.1,287.0$ $\mathrm{Hz}), 140.7,140.2,132.8$ (dd, $J=2.7,2.7 \mathrm{~Hz}), 128.9,128.7$ $(\mathrm{dd}, J=3.4,3.4 \mathrm{~Hz}), 127.5,127.3,127.1,92.1$ (dd, $J=$ 20.2, 14.3 Hz), 34.0, 32.8, 28.2, 27.9, 27.7 (dd, $J=2.4,2.4$ $\mathrm{Hz}), 27.5 ;{ }^{19} \mathrm{~F}$ NMR $\left(471 \mathrm{MHz}, \mathrm{CDCl}_{3}\right) \delta:-91.15(\mathrm{~d}, J=$ $43.5 \mathrm{~Hz}),-91.26(\mathrm{~d}, J=43.4 \mathrm{~Hz})$; ESI-HRMS calcd for $\mathrm{C}_{20} \mathrm{H}_{22} \mathrm{BrF}_{2}(\mathrm{M}+\mathrm{H})^{+}$379.0867, found 379.0865.

4-(3-Cyclohexyl-1,1-difluoroprop-1-en-2-yl)dibenzo$[b, d]$ thiophene $(4 \mathrm{cn})$ : The yield is $98 \%(67.0 \mathrm{mg}$ as colorless oil) after column chromatography. ${ }^{1} \mathrm{H}$ NMR (500 $\left.\mathrm{MHz}, \mathrm{CDCl}_{3}\right) \delta: 8.21 \sim 8.09(\mathrm{~m}, 2 \mathrm{H}), 7.93 \sim 7.80(\mathrm{~m}, 1 \mathrm{H})$, $7.53 \sim 7.43(\mathrm{~m}, 3 \mathrm{H}), 7.38 \sim 7.29(\mathrm{~m}, 1 \mathrm{H}), 2.43(\mathrm{dt}, J=7.2$, $2.3 \mathrm{~Hz}, 2 \mathrm{H}), 1.85 \sim 1.75(\mathrm{~m}, 2 \mathrm{H}), 1.73 \sim 1.59(\mathrm{~m}, 3 \mathrm{H})$, $1.28 \sim 1.20(\mathrm{~m}, 1 \mathrm{H}), 1.19 \sim 1.08(\mathrm{~m}, 3 \mathrm{H}), 1.04 \sim 0.94(\mathrm{~m}$, $2 \mathrm{H}) ;{ }^{13} \mathrm{C} \mathrm{NMR}\left(126 \mathrm{MHz}, \mathrm{CDCl}_{3}\right) \delta: 153.7$ (dd, $J=289.3$, $289.3 \mathrm{~Hz}$ ), 140.0, 139.4, 136.1, 135.9, 129.5 (dd, $J=1.5$, $1.5 \mathrm{~Hz}), 127.6(\mathrm{dd}, J=1.4,1.4 \mathrm{~Hz}), 127.0,124.8,124.5$, $122.9,121.8,121.0,90.4$ (dd, $J=23.9,15.2 \mathrm{~Hz}), 36.1$ (dd, $J=2.6,2.6 \mathrm{~Hz}), 35.8,33.2,26.5,26.2 ;{ }^{19} \mathrm{~F} \mathrm{NMR}(471$ $\left.\mathrm{MHz}, \mathrm{CDCl}_{3}\right) \delta:-86.80(\mathrm{~d}, J=39.2 \mathrm{~Hz}),-91.51(\mathrm{~d}$, $J=39.1 \mathrm{~Hz}$ ); ESI-HRMS calcd for $\mathrm{C}_{21} \mathrm{H}_{20} \mathrm{~F}_{2} \mathrm{SNa}(\mathrm{M}+$ $\mathrm{Na})^{+}$365.1146, found 365.1141.

4-(4-(2-Chlorophenoxy)-1,1-difluoropent-1-en-2-yl)dibenzo $[b, d]$ thiophene (4co): The yield is $68 \%$ (56.6 $\mathrm{mg}$ as colorless oil) after column chromatography. ${ }^{1} \mathrm{H}$ NMR $(500$ $\left.\mathrm{MHz}, \mathrm{CDCl}_{3}\right) \delta: 8.19 \sim 8.07(\mathrm{~m}, 2 \mathrm{H}), 7.88 \sim 7.77(\mathrm{~m}, 1 \mathrm{H})$, $7.51 \sim 7.41(\mathrm{~m}, 3 \mathrm{H}), 7.37 \sim 7.31(\mathrm{~m}, 1 \mathrm{H}), 7.31 \sim 7.27(\mathrm{~m}$, $1 \mathrm{H}), 7.05 \sim 6.98(\mathrm{~m}, 1 \mathrm{H}), 6.86 \sim 6.80(\mathrm{~m}, 1 \mathrm{H}), 6.64 \sim 6.57$ $(\mathrm{m}, 1 \mathrm{H}), 4.39 \sim 4.23(\mathrm{~m}, 1 \mathrm{H}), 3.14 \sim 2.78(\mathrm{~m}, 2 \mathrm{H}), 1.39(\mathrm{~d}$, $J=6.0 \mathrm{~Hz}, 3 \mathrm{H}) ;{ }^{13} \mathrm{C} \mathrm{NMR}\left(126 \mathrm{MHz}, \mathrm{CDCl}_{3}\right) \delta: 154.4$ (dd, $J=291.5,290.2 \mathrm{~Hz}), 153.4,139.9,139.2,136.2,135.8$, 130.5, 128.3 (dd, $J=4.9,1.3 \mathrm{~Hz}), 127.9$ (dd, $J=1.3,1.3$ Hz), 127.5, 127.1, 124.9, 124.6, 124.1, 122.9, 121.9, 121.5, 
$121.3,115.1,88.5(\mathrm{dd}, J=23.2,18.1 \mathrm{~Hz}), 73.2(\mathrm{dd}, J=3.1$, $3.1 \mathrm{~Hz}), 35.0,19.7 ;{ }^{19} \mathrm{~F} \mathrm{NMR}\left(471 \mathrm{MHz}, \mathrm{CDCl}_{3}\right) \delta$ : $-84.91(\mathrm{~d}, J=33.6 \mathrm{~Hz}),-89.52(\mathrm{~d}, J=34.1 \mathrm{~Hz})$; ESIHRMS calcd for $\mathrm{C}_{23} \mathrm{H}_{17} \mathrm{ClF}_{2} \mathrm{OSNa}(\mathrm{M}+\mathrm{Na})^{+} 437.0549$, found 437.0534 .

1-(Benzyloxy)-4-(1,1-difluoro-4,4-dimethylpent-1-en-2yl)benzene (4bp): The yield is $86 \%$ (54.2 $\mathrm{mg}$ as colorless oil) after column chromatography. ${ }^{1} \mathrm{H}$ NMR (500 MHz, $\left.\mathrm{CDCl}_{3}\right) \delta: 7.48 \sim 7.32(\mathrm{~m}, 5 \mathrm{H}), 7.27 \sim 7.22(\mathrm{~m}, 2 \mathrm{H})$, $7.00 \sim 6.92(\mathrm{~m}, 2 \mathrm{H}), 5.07(\mathrm{~s}, 2 \mathrm{H}), 2.38 \sim 2.28(\mathrm{~m}, 2 \mathrm{H})$, 0.83 (s, 6H); $\left.{ }^{13} \mathrm{C} \mathrm{NMR} \mathrm{(126} \mathrm{MHz,} \mathrm{CDCl}_{3}\right) \delta: 157.9,154.5$ (dd, $J=287.4,286.4 \mathrm{~Hz}), 137.1,129.7$ (dd, $J=3.2,2.6$ Hz), 128.7, 128.1, 127.7, 114.8, 90.7 (dd, $J=21.6,12.9$ $\mathrm{Hz}), 70.2,41.3,32.8(\mathrm{dd}, J=2.1,2.1 \mathrm{~Hz}), 29.9 ;{ }^{19} \mathrm{~F}$ NMR $\left(471 \mathrm{MHz}, \mathrm{CDCl}_{3}\right) \delta:-90.64(\mathrm{~d}, J=43.3 \mathrm{~Hz}),-93.17$ (d, $J=43.3 \mathrm{~Hz}$ ); ESI-HRMS calcd for $\mathrm{C}_{20} \mathrm{H}_{22} \mathrm{~F}_{2} \mathrm{ONa}(\mathrm{M}+$ $\mathrm{Na})^{+}$339.1531, found 339.1561.

$N$-(4-(4-(Benzyloxy)phenyl)-5,5-difluoro-1-phenylpent4-en-2-yl)acetamide (4bq): The yield is $60 \%(50.2 \mathrm{mg}$ as colorless oil) after column chromatography. ${ }^{1} \mathrm{H}$ NMR (500 $\left.\mathrm{MHz}, \mathrm{CDCl}_{3}\right) \delta: 7.45 \sim 7.26(\mathrm{~m}, 7 \mathrm{H}), 7.24 \sim 7.16(\mathrm{~m}, 3 \mathrm{H})$, $7.12 \sim 7.07(\mathrm{~m}, 2 \mathrm{H}), 6.98 \sim 6.92(\mathrm{~m}, 2 \mathrm{H}), 5.13 \sim 5.07(\mathrm{~m}$, $1 \mathrm{H}), 5.06(\mathrm{~s}, 2 \mathrm{H}), 4.26 \sim 4.14(\mathrm{~m}, 1 \mathrm{H}), 2.84 \sim 2.70(\mathrm{~m}$, 2H), $2.59 \sim 2.48(\mathrm{~m}, 2 \mathrm{H}), 1.76(\mathrm{~s}, 3 \mathrm{H}) ;{ }^{13} \mathrm{C} \mathrm{NMR}(126$ $\left.\mathrm{MHz}, \mathrm{CDCl}_{3}\right) \delta: 169.7,158.2,154.3(\mathrm{dd}, J=285.9,285.9$ $\mathrm{Hz}), 137.6,137.0,129.6$ (dd, $J=3.2,3.2 \mathrm{~Hz}), 129.3,128.8$, $128.6,128.2,127.6,126.7,125.5(\mathrm{dd}, J=3.7,3.7 \mathrm{~Hz})$, $115.1,89.4$ (dd, $J=21.4,14.9 \mathrm{~Hz}), 70.1,49.3(\mathrm{dd}, J=2.4$, $2.4 \mathrm{~Hz}$ ), 40.3, 32.5, 23.4; ${ }^{19} \mathrm{~F}$ NMR (471 MHz, $\left.\mathrm{CDCl}_{3}\right) \delta$ : $-90.82(\mathrm{~d}, J=43.4 \mathrm{~Hz}),-91.25$ (d, $J=43.4 \mathrm{~Hz}$ ); ESIHRMS calcd for $\mathrm{C}_{26} \mathrm{H}_{25} \mathrm{~F}_{2} \mathrm{NO}_{2} \mathrm{Na}(\mathrm{M}+\mathrm{Na})^{+} 444.1746$, found 444.1754 .

1-(tert-Butyl)-4-(1,1-difluoro-4,4-dimethylpent-1-en-2yl)benzene (4dp): The yield is $84 \%$ ( $44.5 \mathrm{mg}$ as colorless oil) after column chromatography. ${ }^{1} \mathrm{H}$ NMR $(500 \mathrm{MHz}$, $\left.\mathrm{CDCl}_{3}\right) \delta: 7.38 \sim 7.28(\mathrm{~m}, 2 \mathrm{H}), 7.25 \sim 7.19(\mathrm{~m}, 2 \mathrm{H})$, $2.36 \sim 2.27(\mathrm{~m}, 2 \mathrm{H}), 1.31(\mathrm{~s}, 9 \mathrm{H}), 0.80(\mathrm{~s}, 9 \mathrm{H}) ;{ }^{13} \mathrm{C} \mathrm{NMR}$ $\left(126 \mathrm{MHz}, \mathrm{CDCl}_{3}\right) \delta$ : $154.5(\mathrm{dd}, J=287.1,287.1 \mathrm{~Hz})$, $150.0,132.5(\mathrm{dd}, J=2.8,2.4 \mathrm{~Hz}), 128.1(\mathrm{dd}, J=2.7,2.7$ $\mathrm{Hz}), 125.3,90.9$ (dd, $J=34.8,12.6 \mathrm{~Hz}), 41.2,34.6,32.8$, $31.5,29.9 ;{ }^{19} \mathrm{~F}$ NMR $\left(471 \mathrm{MHz}, \mathrm{CDCl}_{3}\right) \delta$ : $-90.10(\mathrm{dd}$, $J=42.6,4.0 \mathrm{~Hz}),-92.70(\mathrm{dd}, J=41.9$, $4.6 \mathrm{~Hz})$; ESIHRMS calcd for $\mathrm{C}_{17} \mathrm{H}_{25} \mathrm{~F}_{2}(\mathrm{M}+\mathrm{H})^{+}$267.1919, found 267.1931. The NMR data were consistent with those reported in previous literature. ${ }^{[12]}$

2-(1,1-Difluoro-4,4-dimethylpent-1-en-2-yl)naphthalene (4ep): The yield is $60 \%$ (31.0 $\mathrm{mg}$ as colorless oil) after column chromatography. ${ }^{1} \mathrm{H}$ NMR $\left(500 \mathrm{MHz}, \mathrm{CDCl}_{3}\right) \delta$ : $7.88 \sim 7.75(\mathrm{~m}, 4 \mathrm{H}), 7.53 \sim 7.42(\mathrm{~m}, 3 \mathrm{H}), 2.47(\mathrm{t}, J=2.5$ $\mathrm{Hz}, 2 \mathrm{H}), 0.84(\mathrm{~s}, 9 \mathrm{H}) ;{ }^{13} \mathrm{C}$ NMR $\left(126 \mathrm{MHz}, \mathrm{CDCl}_{3}\right) \delta$ : 154.8 (dd, $J=290.6,287.8 \mathrm{~Hz}), 133.4,133.2$ (dd, $J=4.8$, $2.9 \mathrm{~Hz}), 132.5,128.0,127.8,127.5(\mathrm{dd}, J=3.0,3.0 \mathrm{~Hz})$, $126.7(\mathrm{dd}, J=2.8,2.8 \mathrm{~Hz}), 126.3,126.1,91.4(\mathrm{dd}, J=21.7$, $12.7 \mathrm{~Hz}), 41.4,33.0$ (dd, $J=2.5,2.5 \mathrm{~Hz}), 29.9$; ${ }^{19} \mathrm{~F}$ NMR $\left(471 \mathrm{MHz}, \mathrm{CDCl}_{3}\right) \delta:-89.24(\mathrm{~d}, J=40.1 \mathrm{~Hz}),-92.03$ $(\mathrm{d}, J=40.2 \mathrm{~Hz})$; ESI-HRMS calcd for $\mathrm{C}_{17} \mathrm{H}_{19} \mathrm{~F}_{2}(\mathrm{M}+\mathrm{H})^{+}$ 261.1449, found 261.1453. The NMR data were consistent with those reported in previous literature. ${ }^{[30]}$

1-(1,1-Difluoro-4,4-dimethylpent-1-en-2-yl)-4-methoxybenzene (4fp): On a $0.2 \mathrm{mmol}$ scale, the yield is $80 \%(38.4$ $\mathrm{mg}$ as colorless oil) after column chromatography; on a 1 mmol scale, the yield is $78 \%$ (188 $\mathrm{mg}$ as colorless oil $)$ after column chromatography. ${ }^{1} \mathrm{H}$ NMR $\left(500 \mathrm{MHz}, \mathrm{CDCl}_{3}\right) \delta$ : $7.24 \sim 7.20(\mathrm{~m}, 2 \mathrm{H}), 6.91 \sim 6.82(\mathrm{~m}, 2 \mathrm{H}), 3.81(\mathrm{~s}, 3 \mathrm{H})$, $2.29(\mathrm{t}, J=2.6 \mathrm{~Hz}, 2 \mathrm{H}), 0.80(\mathrm{~s}, 9 \mathrm{H}) ;{ }^{13} \mathrm{C}$ NMR $(126 \mathrm{MHz}$, $\left.\mathrm{CDCl}_{3}\right) \delta: 158.6(\mathrm{dd}, J=288.9,287.5 \mathrm{~Hz}), 129.7$ (dd, $J=$ 2.8, $2.8 \mathrm{~Hz}), 127.9$ (dd, $J=4.5,2.7 \mathrm{~Hz}), 123.6,113.9,90.8$ (dd, $J=16.1,3.1 \mathrm{~Hz}), 55.4,41.4,32.8(\mathrm{dd}, J=2.4,2.4 \mathrm{~Hz})$, 29.9; ${ }^{19} \mathrm{~F}$ NMR (471 MHz, $\left.\mathrm{CDCl}_{3}\right) \delta:-90.79$ (d, $J=43.4$ $\mathrm{Hz}),-93.32(\mathrm{~d}, J=43.4 \mathrm{~Hz})$; ESI-HRMS calcd for $\mathrm{C}_{14} \mathrm{H}_{18} \mathrm{~F}_{2} \mathrm{O}(\mathrm{M}+\mathrm{H})^{+}$241.1398, found 241.1380. The NMR data were consistent with those reported in previous literature. ${ }^{[12]}$

1-(Benzyloxy)-4-(3-cyclohexyl-1,1-difluoroprop-1-en-2yl)benzene (4bn): The yield is $58 \%$ (39.9 $\mathrm{mg}$ as white solid) after column chromatography. m.p. $94 \sim 96{ }^{\circ} \mathrm{C} ;{ }^{1} \mathrm{H}$ NMR $\left(400 \mathrm{MHz}, \mathrm{CDCl}_{3}\right) \delta: 7.53 \sim 7.31(\mathrm{~m}, 5 \mathrm{H}), 7.26 \sim$ $7.23(\mathrm{~m}, 2 \mathrm{H}), 7.06 \sim 6.92(\mathrm{~m}, 2 \mathrm{H}), 5.08(\mathrm{~s}, 2 \mathrm{H}), 2.25(\mathrm{dt}$, $J=7.3,2.5 \mathrm{~Hz}, 2 \mathrm{H}), 1.75 \sim 1.59(\mathrm{~m}, 5 \mathrm{H}), 1.32 \sim 1.21(\mathrm{~m}$, $1 \mathrm{H}), 1.18 \sim 1.06(\mathrm{~m}, 3 \mathrm{H}), 1.02 \sim 0.87(\mathrm{~m}, 2 \mathrm{H}) ;{ }^{13} \mathrm{C} \mathrm{NMR}$ $\left(101 \mathrm{MHz}, \mathrm{CDCl}_{3}\right) \delta: 157.9,154.0(\mathrm{dd}, J=289.0,285.4$ $\mathrm{Hz}), 137.1,129.5$ (dd, $J=3.2,3.2 \mathrm{~Hz}$ ), 128.8, 128.2, 127.7, $126.7(\mathrm{dd}, J=3.0,3.0 \mathrm{~Hz}), 114.8,90.6(\mathrm{dd}, J=22.0,12.9$ $\mathrm{Hz}), 70.1,35.8$ (dd, $J=2.0,2.0 \mathrm{~Hz}), 35.4,33.0,26.6,26.2$; ${ }^{19} \mathrm{~F}$ NMR $\left(376 \mathrm{MHz}, \mathrm{CDCl}_{3}\right) \delta$ : $-92.18(\mathrm{~d}, J=46.3 \mathrm{~Hz})$, $-92.61\left(\mathrm{~d}, J=46.4 \mathrm{~Hz}\right.$ ); ESI-HRMS calcd for $\mathrm{C}_{22} \mathrm{H}_{24} \mathrm{~F}_{2} \mathrm{O}$ $(\mathrm{M}+\mathrm{H})^{+}$343.1868, found 343.1878. The NMR data were consistent with those reported in previous literature. ${ }^{[21]}$

1-(1,1-Difluoro-4,4-dimethyl-6-phenylhex-1-en-2-yl)-4phenoxybenzene (4gd): The yield is $98 \%(77.1 \mathrm{mg}$ as colorless oil) after column chromatography. ${ }^{1} \mathrm{H}$ NMR (500 $\left.\mathrm{MHz}, \mathrm{CDCl}_{3}\right) \delta: 7.32 \sim 7.27(\mathrm{~m}, 2 \mathrm{H}), 7.26 \sim 7.17(\mathrm{~m}, 4 \mathrm{H})$, $7.14 \sim 7.07(\mathrm{~m}, 2 \mathrm{H}), 7.01 \sim 6.92(\mathrm{~m}, 6 \mathrm{H}), 2.48 \sim 2.40(\mathrm{~m}$, $2 \mathrm{H}), 2.39 \sim 2.34(\mathrm{~m}, 2 \mathrm{H}), 1.45 \sim 1.36(\mathrm{~m}, 2 \mathrm{H}), 0.85(\mathrm{~s}$, $6 \mathrm{H}) ;{ }^{13} \mathrm{C} \mathrm{NMR}\left(126 \mathrm{MHz}, \mathrm{CDCl}_{3}\right) \delta: 157.0,156.5,154.5$ (dd, $J=287.6,287.2 \mathrm{~Hz}), 143.1,130.4$ (dd, $J=4.8,2.5$ $\mathrm{Hz}$ ), 130.0 (dd, $J=2.7,2.7 \mathrm{~Hz}), 129.9,128.4,128.3,125.7$, 123.6, 119.3, 118.6, 90.3 (dd, $J=21.7,13.5 \mathrm{~Hz}), 44.5$, $39.3,35.5$ (dd, $J=2.6,2.6 \mathrm{~Hz}), 30.8,27.6 ;{ }^{19} \mathrm{~F}$ NMR (471 $\left.\mathrm{MHz}, \mathrm{CDCl}_{3}\right) \delta$ : $-89.61(\mathrm{~d}, J=41.6 \mathrm{~Hz}),-92.00(\mathrm{~d}$, $J=41.7 \mathrm{~Hz}$ ); ESI-HRMS calcd for $\mathrm{C}_{26} \mathrm{H}_{26} \mathrm{~F}_{2} \mathrm{O}(\mathrm{M})^{+}$ 392.1952, found 392.1967.

1-Chloro-4-(1,1-difluoro-4,4-dimethylpent-1-en-2-yl)benzene (4hp): The yield is $66 \%$ (32.1 $\mathrm{mg}$ as colorless oil) after column chromatography. ${ }^{1} \mathrm{H}$ NMR $(500 \mathrm{MHz}$, $\left.\mathrm{CDCl}_{3}\right) \delta: 7.36 \sim 7.27(\mathrm{~m}, 2 \mathrm{H}), 7.29 \sim 7.22(\mathrm{~m}, 2 \mathrm{H})$, $2.37 \sim 2.27(\mathrm{~m}, 2 \mathrm{H}), 0.86 \sim 0.75(\mathrm{~m}, 9 \mathrm{H}) ;{ }^{13} \mathrm{C}$ NMR $(126$ $\left.\mathrm{MHz}, \mathrm{CDCl}_{3}\right) \delta: 154.5(\mathrm{dd}, J=290.6,287.8 \mathrm{~Hz}), 134.3$, $132.9,129.9$ (dd, $J=3.1,2.6 \mathrm{~Hz}), 128.7,90.5$ (dd, $J=22.4$, $12.7 \mathrm{~Hz}), 41.3,32.9$ (dd, $J=2.7,2.2 \mathrm{~Hz}$ ), 29.9; ${ }^{19} \mathrm{~F}$ NMR $\left(471 \mathrm{MHz}, \mathrm{CDCl}_{3}\right) \delta:-88.99(\mathrm{dd}, J=39.5,9.0 \mathrm{~Hz})$, $-91.64(\mathrm{dd}, J=39.5,9.0 \mathrm{~Hz})$; ESI-HRMS calcd for $\mathrm{C}_{13} \mathrm{H}_{15} \mathrm{ClF}_{2}(\mathrm{M})^{+}$244.0825, found 244.0851. The NMR data were consistent with those reported in previous litera- 
ture. ${ }^{[12]}$

5-(3-Cyclohexyl-1,1-difluoroprop-1-en-2-yl)-2-methoxypyridine (4in): The yield is $79 \%$ (42.0 mg as colorless oil) after column chromatography. ${ }^{1} \mathrm{H}$ NMR $(500 \mathrm{MHz}$, $\left.\mathrm{CDCl}_{3}\right) \delta: 8.16 \sim 8.05(\mathrm{~m}, 1 \mathrm{H}), 7.53 \sim 7.48(\mathrm{~m}, 1 \mathrm{H})$, $6.78 \sim 6.71(\mathrm{~m}, 1 \mathrm{H}), 3.94(\mathrm{~s}, 3 \mathrm{H}), 2.22(\mathrm{dt}, J=7.2,2.4 \mathrm{~Hz}$, $2 \mathrm{H}), 1.71 \sim 1.60(\mathrm{~m}, 5 \mathrm{H}), 1.31 \sim 1.20(\mathrm{~m}, 1 \mathrm{H}), 1.15 \sim 1.06$ $(\mathrm{m}, 3 \mathrm{H}), 0.99 \sim 0.84(\mathrm{~m}, 2 \mathrm{H}) ;{ }^{13} \mathrm{C} \mathrm{NMR}(126 \mathrm{MHz}$, $\left.\mathrm{CDCl}_{3}\right) \delta: 163.3,154.2(\mathrm{dd}, J=289.9,286.7 \mathrm{~Hz}), 146.4$ (dd, $J=3.6,3.6 \mathrm{~Hz}), 138.6(\mathrm{dd}, J=3.3,3.3 \mathrm{~Hz}), 123.0$ (dd, $J=3.6,3.6 \mathrm{~Hz}), 110.8,88.2(\mathrm{dd}, J=23.7,12.9 \mathrm{~Hz}), 53.6$, $35.8(\mathrm{dd}, J=2.4,2.4 \mathrm{~Hz}), 35.1,33.0,30.3,27.0,26.5,26.2$; ${ }^{19} \mathrm{~F}$ NMR (471 MHz, $\left.\mathrm{CDCl}_{3}\right) \delta$ : $-90.73(\mathrm{~d}, J=44.2 \mathrm{~Hz})$, $-91.50(\mathrm{~d}, J=43.9 \mathrm{~Hz})$; ESI-HRMS calcd for $\mathrm{C}_{15} \mathrm{H}_{20^{-}}$ $\mathrm{F}_{2} \mathrm{NO}(\mathrm{M}+\mathrm{H})^{+}$268.1507, found 268.1513. The NMR data were consistent with those reported in previous literature. ${ }^{[22]}$

(4-(1,1-Difluoro-4,4-dimethyl-6-phenylhex-1-en-2yl)phenyl)(methyl)sulfane (4jd): The yield is $90 \%(62.6$ $\mathrm{mg}$ as colorless oil) after column chromatography. ${ }^{1} \mathrm{H}$ NMR $\left(500 \mathrm{MHz}, \mathrm{CDCl}_{3}\right) \delta: 7.25 \sim 7.13(\mathrm{~m}, 7 \mathrm{H}), 7.01 \sim$ $6.89(\mathrm{~m}, 2 \mathrm{H}), 2.50(\mathrm{~s}, 3 \mathrm{H}), 2.49 \sim 2.44(\mathrm{~m}, 2 \mathrm{H}), 2.41(\mathrm{t}$, $J=2.5 \mathrm{~Hz}, 2 \mathrm{H}), 1.48 \sim 1.41(\mathrm{~m}, 2 \mathrm{H}), 0.88(\mathrm{~s}, 6 \mathrm{H}) ;{ }^{13} \mathrm{C}$ NMR $\left(126 \mathrm{MHz}, \mathrm{CDCl}_{3}\right) \delta: 154.5(\mathrm{dd}, J=289.8,287.9$ $\mathrm{Hz}), 143.1,137.6,132.3$ (dd, $J=2.7,2.7 \mathrm{~Hz}), 129.1$ (dd, $J=2.8,2.8 \mathrm{~Hz}), 128.4,128.3,126.6,125.7,90.4(\mathrm{dd}, J=$ 21.6, $13.4 \mathrm{~Hz}), 44.5,39.0,35.5$ (dd, $J=2.4,2.4 \mathrm{~Hz}), 30.7$, 27.6, $15.8 ;{ }^{19} \mathrm{~F}$ NMR (471 MHz, $\left.\mathrm{CDCl}_{3}\right) \delta$ : $-89.22(\mathrm{~d}$, $J=40.7 \mathrm{~Hz}),-91.65(\mathrm{~d}, J=40.7 \mathrm{~Hz})$; ESI-HRMS calcd for $\mathrm{C}_{21} \mathrm{H}_{25} \mathrm{~F}_{2} \mathrm{~S}(\mathrm{M}+\mathrm{H})^{+}$347.1640, found 347.1646.

2-(1,1-Difluoro-4,4-dimethyl-6-phenylhex-1-en-2-yl)aniline (4kd): The yield is $34 \%(21.2 \mathrm{mg}$ as brown oil) after column chromatography. ${ }^{1} \mathrm{H}$ NMR $(500 \mathrm{MHz}$, $\left.\mathrm{CDCl}_{3}\right) \delta: 7.23 \sim 7.09(\mathrm{~m}, 5 \mathrm{H}), 6.99 \sim 6.86(\mathrm{~m}, 2 \mathrm{H})$, $6.84 \sim 6.67(\mathrm{~m}, 2 \mathrm{H}), 3.57$ (brs, $2 \mathrm{H}), 2.55 \sim 2.23(\mathrm{~m}, 4 \mathrm{H})$, $1.48 \sim 1.41(\mathrm{~m}, 2 \mathrm{H}), 0.93(\mathrm{~s}, 6 \mathrm{H}) ;{ }^{13} \mathrm{C} \mathrm{NMR}(126 \mathrm{MHz}$, $\left.\mathrm{CDCl}_{3}\right) \delta: 154.0(\mathrm{dd}, J=288.6,288.6 \mathrm{~Hz}), 144.1,143.5$, 130.8 (dd, $J=1.0,1.0 \mathrm{~Hz}), 129.1,128.7,128.6,125.9$, $120.7,119.0,116.5,87.7$ (dd, $J=20.9,16.3 \mathrm{~Hz}), 44.6$, $39.2,35.7$ (dd, $J=2.5,2.5 \mathrm{~Hz}), 31.1,27.9 ;{ }^{19} \mathrm{~F}$ NMR (471 $\left.\mathrm{MHz}, \mathrm{CDCl}_{3}\right) \delta:-86.86(\mathrm{~d}, J=37.7 \mathrm{~Hz}),-89.46(\mathrm{~d}$, $J=37.4 \mathrm{~Hz}$ ); ESI-HRMS calcd for $\mathrm{C}_{20} \mathrm{H}_{24} \mathrm{~F}_{2} \mathrm{~N}(\mathrm{M}+\mathrm{H})^{+}$ 316.1871 , found 316.1879 .

$\mathrm{N}$-(3-(3-Cyclohexyl-1,1-difluoroprop-1-en-2-yl)phenyl)methacrylamide (4Ina): The yield is $70 \%(44.5 \mathrm{mg}$ as white solid) after column chromatography. m.p. 88 $90{ }^{\circ} \mathrm{C} ;{ }^{1} \mathrm{H}$ NMR $\left(500 \mathrm{MHz}, \mathrm{CDCl}_{3}\right) \delta: 7.59(\mathrm{~s}, 1 \mathrm{H}), 7.55 \sim$ $7.47(\mathrm{~m}, 2 \mathrm{H}), 7.36 \sim 7.26(\mathrm{~m}, 1 \mathrm{H}), 7.08 \sim 7.03(\mathrm{~m}, 1 \mathrm{H})$, $5.80(\mathrm{~s}, 1 \mathrm{H}), 5.46(\mathrm{~s}, 1 \mathrm{H}), 2.26(\mathrm{dt}, J=7.4,2.5 \mathrm{~Hz}, 2 \mathrm{H})$, $2.06(\mathrm{~s}, 3 \mathrm{H}), 1.71 \sim 1.60(\mathrm{~m}, 5 \mathrm{H}), 1.31 \sim 1.20(\mathrm{~m}, 1 \mathrm{H})$, $1.17 \sim 1.05(\mathrm{~m}, 3 \mathrm{H}), 0.96 \sim 0.85(\mathrm{~m}, 2 \mathrm{H}) ;{ }^{13} \mathrm{C}$ NMR $(126$ $\left.\mathrm{MHz}, \mathrm{CDCl}_{3}\right) \delta: 166.8,154.1(\mathrm{dd}, J=290.1,286.4 \mathrm{~Hz})$, $141.0,138.0,135.2$ (dd, $J=4.6,2.8 \mathrm{~Hz}), 129.1,124.5$ (dd, $J=3.1,3.1 \mathrm{~Hz}), 120.0,120.0(\mathrm{dd}, J=3.4,3.4 \mathrm{~Hz}), 119.0$, $91.1(\mathrm{dd}, J=22.3,12.6 \mathrm{~Hz}), 35.8(\mathrm{dd}, J=2.3,2.3 \mathrm{~Hz})$, 35.3, 33.0, 26.5, 26.2, 18.9; ${ }^{19} \mathrm{~F}$ NMR (471 $\mathrm{MHz}, \mathrm{CDCl}_{3}$ ) $\delta:-90.98(\mathrm{~d}, J=42.8 \mathrm{~Hz}),-91.24(\mathrm{~d}, J=43.1 \mathrm{~Hz})$;
ESI-HRMS calcd for $\mathrm{C}_{19} \mathrm{H}_{24} \mathrm{~F}_{2} \mathrm{NO}(\mathrm{M}+\mathrm{H})^{+} 320.1820$, found 320.1824 .

3-Cyclohexyl- $N$-(3-(3-cyclohexyl-1,1-difluoroprop-1en-2-yl)phenyl)-2-methylpropanamide (4Inb): The yield is $11 \%(8.7 \mathrm{mg}$ as colorless oil) after column chromatography. ${ }^{1} \mathrm{H}$ NMR $\left(500 \mathrm{MHz}, \mathrm{CDCl}_{3}\right) \delta: 7.53 \sim 7.48(\mathrm{~m}, 1 \mathrm{H})$, $7.43(\mathrm{~s}, 1 \mathrm{H}), 7.33 \sim 7.26(\mathrm{~m}, 1 \mathrm{H}), 7.18 \sim 7.09(\mathrm{~m}, 1 \mathrm{H})$, $7.08 \sim 6.98(\mathrm{~m}, 1 \mathrm{H}), 2.50 \sim 2.41(\mathrm{~m}, 1 \mathrm{H}), 2.28 \sim 2.21(\mathrm{~m}$, $2 \mathrm{H}), 1.75 \sim 1.59(\mathrm{~m}, 10 \mathrm{H}), 1.35 \sim 1.07(\mathrm{~m}, 13 \mathrm{H}), 0.96 \sim$ $0.83(\mathrm{~m}, 4 \mathrm{H}) ;{ }^{13} \mathrm{C}$ NMR $\left(126 \mathrm{MHz}, \mathrm{CDCl}_{3}\right) \delta: 175.3,154.1$ (dd, $J=286.8,286.2 \mathrm{~Hz}), 138.3,135.2,129.1,124.3$, $119.7,118.9,91.1(\mathrm{dd}, J=12.3,9.1 \mathrm{~Hz}), 42.1,40.0,35.8$ (dd, $J=2.2,1.5 \mathrm{~Hz}$ ), 35.5, 35.3, 33.7, 33.5, 33.0, 26.7, 26.5, 26.4, 26.3, 26.2, 18.5; ${ }^{19} \mathrm{~F}$ NMR (471 $\mathrm{MHz}, \mathrm{CDCl}_{3}$ ) $\delta$ : $-91.02(\mathrm{~d}, J=43.3 \mathrm{~Hz}),-91.24(\mathrm{~d}, J=43.3 \mathrm{~Hz})$; ESI-HRMS calcd for $\mathrm{C}_{25} \mathrm{H}_{36} \mathrm{~F}_{2} \mathrm{NO}(\mathrm{M}+\mathrm{H})^{+}$404.2759, found 404.2763.

(3a $S, 5 \mathrm{a} R, 8 \mathrm{a} R, 8 \mathrm{~b} S)-3 \mathrm{a}-(3-(4-(B e n z y l o x y) p h e n y l)-4,4-$ difluorobut-3-en-1-yl)-2,2,7,7-tetramethyltetrahydro-5 $\mathrm{H}$ bis([1,3]dioxolo)[4,5-b:4',5'-d]pyran (4br): The yield is $71 \%(71.3 \mathrm{mg}$ as colorless oil) after column chromatography. ${ }^{1} \mathrm{H} \mathrm{NMR}\left(500 \mathrm{MHz}, \mathrm{CDCl}_{3}\right) \delta: 7.46 \sim 7.29(\mathrm{~m}, 5 \mathrm{H})$, $7.30 \sim 7.25(\mathrm{~m}, 2 \mathrm{H}), 6.99 \sim 6.89(\mathrm{~m}, 2 \mathrm{H}), 5.06(\mathrm{~s}, 2 \mathrm{H})$, $4.54(\mathrm{dd}, J=8.0,2.5 \mathrm{~Hz}, 1 \mathrm{H}), 4.24 \sim 4.17(\mathrm{~m}, 1 \mathrm{H}), 4.03(\mathrm{~d}$, $J=2.5 \mathrm{~Hz}, 1 \mathrm{H}), 3.88 \sim 3.79(\mathrm{~m}, 1 \mathrm{H}), 3.73(\mathrm{~d}, J=12.9 \mathrm{~Hz}$, $1 \mathrm{H}), 2.80 \sim 2.56(\mathrm{~m}, 2 \mathrm{H}), 1.93(\mathrm{td}, J=13.0,4.7 \mathrm{~Hz}, 1 \mathrm{H})$, $1.73(\mathrm{td}, J=13.0,4.7 \mathrm{~Hz}, 1 \mathrm{H}), 1.52(\mathrm{~s}, 3 \mathrm{H}), 1.39(\mathrm{~s}, 3 \mathrm{H})$, $1.33(\mathrm{~s}, 3 \mathrm{H}), 1.29(\mathrm{~s}, 3 \mathrm{H}) ;{ }^{13} \mathrm{C} \mathrm{NMR}\left(126 \mathrm{MHz}, \mathrm{CDCl}_{3}\right) \delta$ : $157.9,153.4(\mathrm{dd}, J=287.5,287.1 \mathrm{~Hz}), 137.0,129.3$ (dd, $J=3.7,3.7 \mathrm{~Hz}), 128.7,128.1,127.5,125.9$ (dd, $J=2.1,1.7$ Hz), 114.9, 109.1, 107.7, 103.7, 91.5 (dd, $J=19.8,14.8$ $\mathrm{Hz}), 73.9,70.9,70.7,70.1,61.1,39.4$ (dd, $J=2.6,2.6 \mathrm{~Hz}$ ), 26.5, 25.9, 25.1, 24.3, 21.4; ${ }^{19} \mathrm{~F}$ NMR (471 $\mathrm{MHz}, \mathrm{CDCl}_{3}$ ) $\delta:-91.77(\mathrm{~d}, J=46.0 \mathrm{~Hz}),-91.89(\mathrm{~d}, J=45.0 \mathrm{~Hz})$; ESI-HRMS calcd for $\mathrm{C}_{28} \mathrm{H}_{32} \mathrm{~F}_{2} \mathrm{O}_{6} \mathrm{Na}(\mathrm{M}+\mathrm{Na})^{+}$525.2059, found 525.2059.

Methyl (S)-5-(4-(benzyloxy)phenyl)-2-((tert-butoxycarbonyl)amino)-6,6-difluorohex-5-enoate (4bs): The yield is $63 \%$ (58.5 mg as colorless oil) after column chromatography. ${ }^{1} \mathrm{H}$ NMR $\left(500 \mathrm{MHz}, \mathrm{CDCl}_{3}\right) \delta: 7.46 \sim 7.29(\mathrm{~m}, 5 \mathrm{H})$, $7.23 \sim 7.17(\mathrm{~m}, 2 \mathrm{H}), 7.00 \sim 6.90(\mathrm{~m}, 2 \mathrm{H}), 5.06(\mathrm{~s}, 2 \mathrm{H})$, $5.04 \sim 4.93(\mathrm{~m}, 1 \mathrm{H}), 4.39 \sim 4.20(\mathrm{~m}, 1 \mathrm{H}), 3.70(\mathrm{~s}, 3 \mathrm{H})$, $2.52 \sim 2.35(\mathrm{~m}, 2 \mathrm{H}), 1.99 \sim 1.79(\mathrm{~m}, 1 \mathrm{H}), 1.69 \sim 1.60(\mathrm{~m}$, 1H), $1.45(\mathrm{~s}, 9 \mathrm{H}) ;{ }^{13} \mathrm{C} \mathrm{NMR}\left(126 \mathrm{MHz}, \mathrm{CDCl}_{3}\right) \delta: 173.1$, $158.2,155.5,153.6(\mathrm{dd}, J=288.6,288.6 \mathrm{~Hz}), 136.9,129.4$ (dd, $J=3.2,3.2 \mathrm{~Hz}$ ), 128.7, 128.2, 127.6, 125.4, 115.0, 90.8 (dd, $J=17.9,17.9 \mathrm{~Hz}$ ), 80.1, 70.2, 53.1, 52.4, 30.9, 28.4, 24.0; ${ }^{19} \mathrm{~F}$ NMR (471 MHz, $\left.\mathrm{CDCl}_{3}\right) \delta$ : -91.43; ESIHRMS calcd for $\mathrm{C}_{25} \mathrm{H}_{29} \mathrm{~F}_{2} \mathrm{NO}_{5} \mathrm{Na}(\mathrm{M}+\mathrm{Na})^{+}$484.1906, found 484.1906.

$(8 R, 9 S, 10 S, 13 R, 14 S, 17 R)-17-((R)-6-(4-(B e n z y l o x y)-$ phenyl)-7,7-difluorohept-6-en-2-yl)-10,13-dimethyldodecahydro-3H-cyclopenta $[a]$ phenanthrene$3,7,12(2 H, 4 H)$-trione (4bt): The yield is $36 \%(44.2 \mathrm{mg}$ as white solid) after column chromatography. m.p. 174 $176{ }^{\circ} \mathrm{C} ;{ }^{1} \mathrm{H}$ NMR $\left(500 \mathrm{MHz}, \mathrm{CDCl}_{3}\right) \delta: 7.46 \sim 7.30(\mathrm{~m}$, $5 \mathrm{H}), 7.24 \sim 7.16(\mathrm{~m}, 2 \mathrm{H}), 7.02 \sim 6.89(\mathrm{~m}, 2 \mathrm{H}), 5.07(\mathrm{~s}$, 
$2 \mathrm{H}), 2.95 \sim 2.78(\mathrm{~m}, 3 \mathrm{H}), 2.37 \sim 1.92(\mathrm{~m}, 15 \mathrm{H}), 1.87 \sim$ $1.75(\mathrm{~m}, 1 \mathrm{H}), 1.67 \sim 1.55(\mathrm{~m}, 2 \mathrm{H}), 1.39(\mathrm{~s}, 3 \mathrm{H}), 1.28 \sim$ $1.18(\mathrm{~m}, 5 \mathrm{H}), 1.04(\mathrm{~s}, 3 \mathrm{H}), 0.77(\mathrm{~d}, J=6.6 \mathrm{~Hz}, 3 \mathrm{H}) ;{ }^{13} \mathrm{C}$ NMR (126 MHz, $\left.\mathrm{CDCl}_{3}\right) \delta: 212.2,209.2,208.9,158.0$, 155.9 (dd, $J=286.5,266.7 \mathrm{~Hz}$ ), 137.1, 129.5 (dd, $J=3.2$, $3.2 \mathrm{~Hz}), 128.8,128.2,127.7,126.4(\mathrm{dd}, J=2.4,2.4 \mathrm{~Hz})$, $114.9,92.0(\mathrm{dd}, J=19.7,15.8 \mathrm{~Hz}), 70.2,57.0,51.9,49.2$, $47.0,45.9,45.7,45.1,42.9,38.8,36.6,36.2,35.9,35.4$, $34.8,28.1,27.9,25.3,24.7,22.1,19.1,12.0 ;{ }^{19} \mathrm{~F}$ NMR $\left(471 \mathrm{MHz}, \mathrm{CDCl}_{3}\right) \delta:-92.71(\mathrm{~d}, J=46.3 \mathrm{~Hz}),-92.83$ (d, $J=46.8 \mathrm{~Hz}$ ); ESI-HRMS calcd for $\mathrm{C}_{39} \mathrm{H}_{46} \mathrm{~F}_{2} \mathrm{O}_{4} \mathrm{Na}(\mathrm{M}+$ $\mathrm{Na})^{+}$639.3256, found 639.3262.

\subsection{Mechanism experiments}

Using the general procedure for Tables 2 and 3, (bromomethyl)cyclopropane (2u) and 6-iodohex-1-ene (2v) were used to participate in the coupling reaction. After the reaction, the mixture was purified by flash column chromatography to afford the crude product. In the case of (bromomethyl)cyclopropane (2u), the crude NMR spectra showed that a large amount of recovered 1 b $(54 \%$ NMR yield determined by ${ }^{19} \mathrm{~F}$ NMR analysis, benzotrifluoride was used as an internal standard) and the ring-opening product $4 \mathrm{bu}$ were obtained $(25 \%$ NMR yield determined by ${ }^{19} \mathrm{~F}$ NMR analysis, benzotrifluoride was used as an internal standard). Despite the complexity of the spectrum, the presence of new vinyl protons and their splitting patterns confirmed the formation of ring-opening products. In the case of 6-iodohex-1-ene (2v), a mixture of cyclized product 4 bva and linear acyclic product 4 bvb was obtained. Despite the complexity of the spectrum, the presence of vinyl protons and their splitting patterns confirmed the linear acyclic product $\mathbf{4 b v b}$. Using the general procedure for Tables 2 and 3 , cyclized product 4 bva could be synthesized as $\mathbf{4 b w}$ from $\mathbf{1 b}$ and (iodomethyl)cyclopentane (2w). The obtained standard product was used in the gas chromatography confirmation. The total yield of mixture products 4 bva and $\mathbf{4 b v b}$ is $61 \%$ (determined by ${ }^{19} \mathrm{~F}$ NMR analysis, benzotrifluoride was used as an internal standard), $n$ (4bva) $: n$ (4bvb) $=5: 1$ (determined by ${ }^{19} \mathrm{~F}$ NMR and GC). GC retention time of $\mathbf{4 b v a}$ is $9.98 \mathrm{~min}$, while 4bvb is $9.35 \mathrm{~min}$.

1-(Benzyloxy)-4-(4-cyclopentyl-1,1-difluorobut-1-en-2yl)benzene (4bw, standard product of $4 \mathbf{b v a}$ ): The yield is $31 \%$ (21.3 mg as colorless oil) after column chromatography. ${ }^{1} \mathrm{H} \mathrm{NMR}\left(500 \mathrm{MHz}, \mathrm{CDCl}_{3}\right) \delta: 7.48 \sim 7.30(\mathrm{~m}, 5 \mathrm{H})$, $7.26 \sim 7.20(\mathrm{~m}, 2 \mathrm{H}), 7.01 \sim 6.94(\mathrm{~m}, 2 \mathrm{H}), 5.07(\mathrm{~s}, 2 \mathrm{H})$, $2.37(\mathrm{tt}, J=8.1,2.5 \mathrm{~Hz}, 2 \mathrm{H}), 1.81 \sim 1.69(\mathrm{~m}, 3 \mathrm{H}), 1.64 \sim$ $1.45(\mathrm{~m}, 4 \mathrm{H}), 1.40 \sim 1.33(\mathrm{~m}, 2 \mathrm{H}), 1.12 \sim 1.00(\mathrm{~m}, 2 \mathrm{H})$; ${ }^{13} \mathrm{C}$ NMR $\left(126 \mathrm{MHz}, \mathrm{CDCl}_{3}\right) \delta: 158.0,153.5$ (dd, $J=$ $287.5,287.5 \mathrm{~Hz}), 137.1,129.5$ (dd, $J=3.4,3.4 \mathrm{~Hz}), 128.8$, $128.2,127.6,126.5,114.9,92.2(\mathrm{dd}, J=17.2,17.2 \mathrm{~Hz})$, $70.2,39.8,34.4$ (dd, $J=2.5,2.5 \mathrm{~Hz}), 32.6,27.1,25.3 ;{ }^{19} \mathrm{~F}$ NMR (471 MHz, $\left.\mathrm{CDCl}_{3}\right) \delta$ : -92.90 ; ESI-HRMS calcd for $\mathrm{C}_{22} \mathrm{H}_{24} \mathrm{~F}_{2} \mathrm{O}(\mathrm{M}+\mathrm{H})^{+}$343.1868, found 343.1868.

Using the general procedure for Tables 2 and 3, a radical inhibitor TEMPO was added to the standard conditions; this reaction has been significantly inhibited in the presence of 0.5 equiv. of TEMPO. After adding 3.0 equiv. of TEMPO, the reaction was completely shut down. And the $\mathrm{R}-\mathrm{TEMPO}$ was also isolated.

2,2,6,6-Tetramethyl-1-(3-(naphthalen-2-yloxy)propoxy)piperidine (R-TEMPO): The product was isolated by column chromatography $(25.9 \mathrm{mg}, 0.076 \mathrm{mmol}$, as colorless oil). ${ }^{1} \mathrm{H}$ NMR (500 MHz, $\left.\mathrm{CDCl}_{3}\right) \delta: 7.79 \sim 7.71(\mathrm{~m}, 3 \mathrm{H})$, $7.47 \sim 7.42(\mathrm{~m}, 1 \mathrm{H}), 7.36 \sim 7.31(\mathrm{~m}, 1 \mathrm{H}), 7.18 \sim 7.14(\mathrm{~m}$, $2 \mathrm{H}), 4.22(\mathrm{t}, J=6.5 \mathrm{~Hz}, 2 \mathrm{H}), 3.98(\mathrm{t}, J=6.2 \mathrm{~Hz}, 2 \mathrm{H})$, $2.16 \sim 2.03(\mathrm{~m}, 2 \mathrm{H}), 1.64 \sim 1.30(\mathrm{~m}, 6 \mathrm{H}), 1.19(\mathrm{~s}, 6 \mathrm{H})$, $1.12(\mathrm{~s}, 6 \mathrm{H}) ;{ }^{13} \mathrm{C}$ NMR $\left(126 \mathrm{MHz}, \mathrm{CDCl}_{3}\right) \delta: 157.1,134.8$, $129.4,129.1,127.8,126.9,126.4,123.6,119.2,106.8$, 72.9, 65.2, 59.9, 39.8, 33.2, 28.9, 20.3, 17.3; ESI-HRMS calcd for $\mathrm{C}_{22} \mathrm{H}_{32} \mathrm{NO}_{2}(\mathrm{M}+\mathrm{H})^{+} 342.2428$, found 342.2435.

Supporting Information The NMR spectra of reported new compounds are available free of charge via the Internet at http://sioc-journal.cn/.

\section{References}

[1] (a) Zhang, X.; Cao, S. Tetrahedron Lett. 2017, 58, 375.

(b) Du, B.; Chan, C.-M.; Lee, P.-Y.; Cheung, L.-H.; Xu, X.; Lin, Z.; Yu, W.-Y. Nat. Commun. 2021, 12, 412.

(c) Zhang, X.-J.; Cheng, Y.-M.; Zhao, X.-W.; Cao, Z.-Y.; Xiao, X.; Xu, Y. Org. Chem. Front. 2021, 8, 2315.

(d) Zhuo, K.-F.; Xu, W.-Y.; Gong, T.-J.; Fu, Y. Chem. Commun. 2020, 56, 2340.

(e) Ma, X.; Song, Q. Chem. Soc. Rev. $2020,49,9197$.

(f) Sheng, J.; Wu, N.; Liu, X.; Liu, F.; Liu, S.; Ding, W.; Liu, C.; Cheng, X. Chin. J. Org. Chem. 2020, 40, 3873 (in Chinese).

(盛杰, 吴娜, 刘旭, 刘峰, 刘帅, 丁伟杰, 刘畅, 程旭, 有机化 学, 2020, 40, 3873.)

(g) Shen, X.; Liu, Q.; Ni, C.; Hu, J. Chin. J. Chem. 2014, 32, 703.

(h) Xiong, B.; Wang, T.; Sun, H.; Li, Y.; Kramer, S.; Cheng, G.-J.; Lian, Z. ACS Catal. 2020, 10, 13616.

[2] (a) Fujita, T.; Fuchibe, K.; Ichikawa, J. Angew. Chem., Int. Ed. 2018, 58, 390 .

(b) Cai, Y.; Tan, D.; Zhang, Q.; Lü, W.; Li, Q.; Wang, H. Chin. Chem. Lett. 2021, 32, 417.

[3] (a) He, S.-J.; Pi, J.-J.; Li, Y.; Lu, X.; Fu, Y. Acta Chim. Sinica 2018 , 76, 956 (in Chinese).

(何世江, 皮静静, 李炎, 陆喜, 傅尧, 化学学报, 2018, 76, 956.) (b) Song, S.; Liu, H.; Wang, L.; Zhu, C.; Loh, T. P.; Feng, C. Chin. J. Chem. 2019, 37, 1036.

(c) Gao, P.; Wang, G.; Xi, L.; Wang, M.; Li, S.; Shi, Z. Chin. J. Chem. 2019, 37, 1009.

(d) Zhang, J.; Wang, B.; Liu, Y.; Cao, S. Chin. J. Org. Chem. 2019, 39, 249 (in Chinese).

(张娟, 王碧云, 刘熠森, 曹松, 有机化学, 2019, 39, 249.)

(e) He, S.-Y.; Zhang, X.-G. Org. Chem. Front. 2020, 7, 3174.

[4] (a) Hu, X.-S.; Ding, P.-G.; Yu, J.-S.; Zhou, J. Org. Chem. Front. 2019, 6, 2500 .

(b) Huang, X.; Zhao, W.; Liang, Y.; Wang, M.; Zhan, Y.; Zhang, Y.; Kong, L.; Wang, Z.-X.; Peng, B. Org. Chem. Front. 2021, 8, 1280.

[5] (a) Lin, T.-Y.; Pan, Z.; Tu, Y.; Zhu, S.; Wu, H.-H.; Liu, Y.; Li, Z.; Zhang, J. Angew. Chem., Int. Ed. 2020, 59, 22957.

(b) Hu, J.; Yang, Y.; Lou, Z.; Ni, C.; Hu, J. Chin. J. Chem. 2018, 36, 1202.

[6] Magueur, G.; Crousse, B.; Ourévitch, M.; Bonnet-Delpon, D.; Bégué, J.-P. J. Fluorine Chem. 2006, 127, 637.

[7] (a) Qin, W.; Chen, J.; Xiong, W.; Liu, G. Chin. J. Org. Chem. 2020, 40, 3177 (in Chinese). 
(秦文兵, 陈嘉怡, 熊威, 刘国凯, 有机化学, 2020, 40, 3177.)

(b) Jin, Y.; Wang, Y.; Bao, K.; Sheng, R.; Tao, X. Chin. J. Org. Chem. 2019, 39, 2726 (in Chinese).

(陶雪芬, 盛荣, 鲍㫃, 王玉新, 金银秀, 有机化学, 2019, 39, 2726.)

(c) Xie, Q.; Hu, J. Chin. J. Chem. 2020, 38, 202.

[8] (a) Zhao, Y.; Huang, W.; Zhu, L.; Hu, J. Org. Lett. 2010, 12, 1444. (b) Hu, M.; He, Z.; Gao, B.; Li, L.; Ni, C.; Hu, J. J. Am. Chem. Soc. 2013, 135,17302

(c) Hu, M.; Ni, C.; Li, L.; Han, Y.; Hu, J. J. Am. Chem. Soc. 2015, 137,14496

[9] (a) Zheng, J.; Cai, J.; Lin, J.-H.; Guo, Y.; Xiao, J.-C. Chem. Commun. 2013, 49, 7513.

(b) Yu, J.; Lin, J.; Xiao, J. Chin. J. Org. Chem. 2019, 39, 265 (in Chinese). (于蛟, 林锦鸿, 肖吉昌, 有机化学, 2019, 39, 265.)

[10] Zhang, Z.; Yu, W.; Wu, C.; Wang, C.; Zhang, Y.; Wang, J. Angew. Chem., Int. Ed. 2016, 55, 273.

[11] (a) Zeng, H.; Cai, Y.; Jiang, H.; Zhu, C. Org. Lett. 2021, 23, 66. (b) Liu, Y.; Zhou, Y.; Zhao, Y.; Qu, J. Org. Lett. 2017, 19, 946.

(c) Zeng, H.; Zhu, C.; Jiang, H. Org. Lett. 2019, 21, 1130.

(d) Cai, Y.; Zeng, H.; Zhu, C.; Liu, C.; Liu, G.; Jiang, H. Org. Chem. Front. 2020, 7, 1260.

(e) Wu, X.; Xie, F.; Gridnev, I. D.; Zhang, W. Org. Lett. 2018, 20, 1638 .

(f) Gao, P.; Yuan, C.; Zhao, Y.; Shi, Z. Chem 2018, 4, 2201.

(g) Gao, P.; Gao, L.; Xi, L.; Zhang, Z.; Li, S.; Shi, Z. Org. Chem. Front. 2020, 7, 2618.

(h) Chen, G.; Wang, L.; Liu, X.; Liu, P. Adv. Synth. Catal. 2020, 362, 2990.

(i) Yao, C.; Wang, S.; Norton, J.; Hammond, M. J. Am. Chem. Soc. 2020, 142, 4793.

(j) Chen, F.; Xu, X.; He, Y.; Huang, G.; Zhu, S. Angew. Chem., Int. Ed. 2020, 59, 5398.

(k) Fuchibe, K.; Hatta, H.; Oh, K.; Oki, R.; Ichikawa, J. Angew. Chem., Int. Ed. 2017, 56, 5890.

(1) Fuchibe, K.; Takahashi, M.; Ichikawa, J. Angew. Chem., Int. Ed. 2012, 51, 12059.

(m) Ichitsuka, T.; Fujita, T.; Arita, T.; Ichikawa, J. Angew. Chem., Int. Ed. 2014, 53, 7564.

(n) Ichitsuka, T.; Fujita, T.; Ichikawa, J. ACS Catal. 2015, 5, 5947.

(o) Jaroschik, F. Chem. Eur. J. 2018, 24, 14572.

(p) Kojima, R.; Akiyama, S.; Ito, H. Angew. Chem., Int. Ed. 2018, 57, 7196 .

(q) Kojima, R.; Kubota, K.; Ito, H. Chem. Commun. 2017, 53, 10688.

(r) Kojima, Y.; Takata, T.; Hirano, K.; Miura, M. Chem. Lett. 2020, $49,637$.

(s) Liu, Y.; Li, C.; Liu, C.; He, J.; Zhao, X.; Cao, S. Tetrahedron Lett. 2020, 61, 151940.

(t) Liu, Z.; Tu, X.-S.; Guo, L.-T.; Wang, X.-C. Chem. Sci. 2020, 11, 11548.

(u) Yan, S.-S.; Wu, D.-S.; Ye, J.-H.; Gong, L.; Zeng, X.; Ran, C.-K.; Gui, Y.-Y.; Li, J.; Yu, D.-G. ACS Catal. 2019, 9, 6987.

(v) Pan, Q.; Ping, Y.; Wang, Y.; Guo, Y.; Kong, W. J. Am. Chem. Soc. 2021, 143, 10282.

(w) Tian, F.; Yan, G.; Yu, J. Chem. Commun. 2019, 55, 13486.

(x) Xie, S.-L.; Cui, X.-Y.; Gao, X.-T.; Zhou, F.; Wu, H.-H.; Zhou, J. Org. Chem. Front. 2019, 6, 3678.

(y) Li, Y.; Wang, Y.; Zhu, L.; Qu, L.; Lan, Y. Chin. J. Org. Chem. 2019, 39, 38 (in Chinese).

(李园园, 王元鉴, 朱碟, 屈凌波, 蓝宇, 有机化学, 2019, 39, 38.)

[12] Dai, W.; Lin, Y.; Wan, Y.; Cao, S. Org. Chem. Front. 2018, 5, 55.

[13] Wang, M.; Pu, X.; Zhao, Y.; Wang, P.; Li, Z.; Zhu, C.; Shi, Z. J. Am. Chem. Soc. 2018, 140, 9061.

[14] Miura, T.; Ito, Y.; Murakami, M. Chem. Lett. 2008, 37, 1006.

[15] Huang, Y.; Hayashi, T. J. Am. Chem. Soc. 2016, 138, 12340.

[16] Cheng, R.; Xu, C.; Zhang, X. Chin. J. Org. Chem. 2020, 40, 3307 (in Chinese).

(程然, 徐畅, 张新刚, 有机化学, 2020, 40, 3307.)

[17] (a) Phelan, J. P.; Wiles, R. J.; Lang, S. B.; Kelly, C. B.; Molander, G. A. Chem. Sci. 2018, 9, 3215.

(b) Lang, S. B.; Wiles, R. J.; Kelly, C. B.; Molander, G. A. Angew. Chem., Int. Ed. 2017, 56, 15073.

(c) Wiles, R. J.; Phelan, J. P.; Molander, G. A. Chem. Commun. 2019, 55, 7599 .

[18] (a) Chen, H.; Anand, D.; Zhou, L. Asian J. Org. Chem. 2019, 8, 661 .

(b) Li, L.; Xiao, T.; Chen, H.; Zhou, L. Chem. Eur. J. 2017, 23, 2249.

(c) Anand, D.; Sun, Z.; Zhou, L. Org. Lett. 2020, 22, 2371.

(d) Chen, H.; Xiao, T.; Li, L.; Anand, D.; He, Y.; Zhou, L. $A d v$ Synth. Catal. 2017, 359, 3642.

(e) He, Y.; Anand, D.; Sun, Z.; Zhou, L. Org. Lett. 2019, 21, 3769.

(f) Xiao, T.; Li, L.; Zhou, L. J. Org. Chem. 2016, 81, 7908.

[19] (a) Guo, Y.-Q.; Wang, R.; Song, H.; Liu, Y.; Wang, Q. Org. Lett. 2020, 22, 709

(b) Guo, Y.-Q.; Wu, Y.; Wang, R.; Song, H.; Liu, Y.; Wang, Q. Org. Lett. 2021, 23, 2353.

(c) Xiang, P.; He, L.; Li, H.; Qi, Z.; Zhang, M.; Fu, Q.; Wei, J.; Du, X.; Yi, D.; Wei, S. Tetrahedron Lett. 2020, 61, 152369.

[20] Lu, X.; Wang, Y.; Zhang, B.; Pi, J.-J.; Wang, X.-X.; Gong, T.-J.; Xiao, B.; Fu, Y. J. Am. Chem. Soc. 2017, 139, 12632.

[21] Lu, X.; Wang, X.-X.; Gong, T.-J.; Pi, J.-J.; He, S.-J.; Fu, Y. Chem. Sci. 2019, 10, 809.

[22] Lan, Y.; Yang, F.; Wang, C. ACS Catal. 2018, 8, 9245.

[23] (a) Yu, L.; Tang, M.-L.; Si, C.-M.; Meng, Z.; Liang, Y.; Han, J.; Sun, X. Org. Lett. 2018, 20, 4579.

(b) Du, H.-W.; Chen, Y.; Sun, J.; Gao, Q.-S.; Wang, H.; Zhou, M.-D. S. Org. Lett. 2020, 22, 9342.

(c) Li, Z.; Wang, K.-F.; Zhao, X.; Ti, H.; Liu, X.-G.; Wang, H. Nat. Commun. 2020, 11, 5036.

(d) Lin, Z.; Lan, Y.; Wang, C. Org. Lett. 2019, 21, 8316.

[24] (a) Ding, D.; Lan, Y.; Lin, Z.; Wang, C. Org. Lett. 2019, 21, 2723.

(b) Lin, Z.; Lan, Y.; Wang, C. Org. Lett. 2020, 22, 3509.

(c) Lu, X.-Y.; Jiang, R.-C.; Li, J.-M.; Liu, C.-C.; Wang, Q.-Q.; Zhou, H.-P. Org. Biomol. Chem. 2020, 18, 3674.

(d) Fan, P.; Zhang, C.; Lan, Y.; Lin, Z.; Zhang, L.; Wang, C. Chem. Commun. 2019, 55, 12691.

(e) Jin, Y.; Wu, J.; Lin, Z.; Lan, Y.; Wang, C. Org. Lett. 2020, 22, 5347.

(f) Zhu, C.; Liu, Z.-Y.; Tang, L.; Zhang, H.; Zhang, Y.-F.; Walsh, P. J.; Feng, C. Nat. Commun. 2020, 11, 4860.

[25] (a) Kawamata, Y.; Vantourout, J. C.; Hickey, D. P.; Bai, P.; Chen, L.; Hou, Q.; Qiao, W.; Barman, K.; Edwards, M. A.; Garrido-Castro, A. F.; deGruyter, J. N.; Nakamura, H.; Knouse, K.; Qin, C.; Clay, K. J.; Bao, D.; Li, C.; Starr, J. T.; Garcia-Irizarry, C.; Sach, N.; White, H. S.; Neurock, M.; Minteer, S. D.; Baran, P. S. J. Am. Chem. Soc. 2019, 141, 6392.

(b) Kim, H.; Kim, H.; Lambert, T. H.; Lin, S. J. Am. Chem. Soc. 2020, 142, 2087.

(c) Li, C.; Kawamata, Y.; Nakamura, H.; Vantourout, J. C.; Liu, Z.; Hou, Q.; Bao, D.; Starr, J. T.; Chen, J.; Yan, M.; Baran, P. S. Angew. Chem., Int. Ed. 2017, 56, 13088.

(d) Wang, P.; Yang, Z.; Wang, Z.; Xu, C.; Huang, L.; Wang, S.; Zhang, H.; Lei, A. Angew. Chem., Int. Ed. 2019, 58, 15747.

(e) Liu, Q.; Sun, B.; Liu, Z.; Kao, Y.; Dong, B.-W.; Jiang, S.-D.; Li, F.; Liu, G.; Yang, Y.; Mo, F. Chem. Sci. 2018, 9, 8731.

(f) Liu, D.; Ma, H.-X.; Fang, P.; Mei, T.-S. Angew. Chem., Int. Ed. 2019, 58, 5033.

(g) Zou, Z.; Zhang, W.; Wang, Y.; Pan, Y. Org. Chem. Front. 2021, 8,2786 .

(h) Lu, L.; Li, H.; Zheng, Y.; Bu, F.; Lei, A. CCS Chem. 2020, 2, 2669.

(i) Dou, G.-Y.; Jiang, Y.-Y.; Xu, K.; Zeng, C.-C. Org. Chem. Front. 2019, 6, 2392. 
(j) Liang, K.; Wang, S.; Cong, H.; Lu, L.; Lei, A. CCS Chem. 2021, 3, 1727 .

(k) Huang, C.; Xu, H.-C. Sci. China Chem. 2019, 62, 1501.

(1) Feng, E.; Hou, Z.; Xu, H. Chin. J. Org. Chem. 2019, 39, 1424 (in Chinese).

(冯恩祺，侯中伟，徐海超，有机化学， 2019, 39, 1424.)

(m) Zhang, C.; Bu, F.; Zeng, C.; Wang, D.; Lu, L.; Zhang, H.; Lei, A. CCS Chem. 2021, 3, 1404.

(n) Deng, Y.; Lu, F.; You, S.; Xia, T.; Zheng, Y.; Lu, C.; Yang, G.; Chen, Z.; Gao, M.; Lei, A. Chin. J. Chem. 2019, 37, 817.

(o) Hou, Z. W.; Xu, H. C. Chin. J. Chem. 2020, 38, 394.

(p) Liu, S.; Zhao, W.; Li, J.; Wu, N.; Liu, C.; Wang, X.; Li, S.; Zhu, Y.; Liang, Y.; Cheng, X. CCS Chem. 2021, 3, 872.

(q) Qiu, Y.; Zhu, C.; Stangier, M.; Struwe, J.; Ackermann, L. CCS Chem. 2020, 2, 1529.

(r) Xu, Y.; Lin, L.; Han, Y.; Liu, Y. Chin. J. Org. Chem. 2021, 41, 934 (in Chinese).

(许颖，林立青，韩莹徽，刘颖杰，有机化学，2021，41,934.)

(s) Yang, Q.-L.; Wang, X.-Y.; Weng, X.-J.; Yang, X.; Xu, X.-T.; Tong, X.; Fang, P.; Wu, X.-Y.; Mei, T.-S. Acta Chim. Sinica 2019, 77,866 (in Chinese).

(杨启亮, 王向阳, 翁信军, 杨祥, 徐学涛, 童晓峰, 方萍, 伍新 燕, 梅天胜, 化学学报, 2019, 77, 866.)

(t) Zhang, Q.; Liang, K.; Guo, C. CCS Chem. 2021, 3, 338.

[26] (a) Li, H.; Breen, C. P.; Seo, H.; Jamison, T. F.; Fang, Y.-Q.; Bio, M. M. Org. Lett. 2018, 20, 1338.

(b) Jiao, K.-J.; Li, Z.-M.; Xu, X.-T.; Zhang, L.-P.; Li, Y.-Q.; Zhang, K.; Mei, T.-S. Org. Chem. Front. 2018, 5, 2244.

(c) Lai, Y.-L.; Huang, J.-M. Org. Lett. 2017, 19, 2022.

(d) Qiu, H.; Shuai, B.; Wang, Y.-Z.; Liu, D.; Chen, Y.-G.; Gao,
P.-S.; Ma, H.-X.; Chen, S.; Mei, T.-S. J. Am. Chem. Soc. 2020, 142, 9872.

[27] (a) Chen, X.; Luo, X.; Peng, X.; Guo, J.; Zai, J.; Wang, P. Chem. Eur. J. 2020, 26, 3226.

(b) Gomes, P.; Gosmini, C.; Périchon, J. J. Org. Chem. 2003, 68, 1142.

(c) Gomes, P.; Gosmini, C.; Périchon, J. Tetrahedron 2003, 59, 2999.

(d) Jiao, K.-J.; Liu, D.; Ma, H.-X.; Qiu, H.; Fang, P.; Mei, T.-S. Angew. Chem., Int. Ed. 2020, 59, 6520.

(e) Kumar, G. S.; Peshkov, A.; Brzozowska, A.; Nikolaienko, P.; Zhu, C.; Rueping, M. Angew. Chem., Int. Ed. 2020, 59, 6513.

(f) Wang, X.-X.; Lu, X.; Li, Y.; Wang, J.-W.; Fu, Y. Sci. China Chem. 2020, 63, 1586.

(g) Truesdell, B. L.; Hamby, T. B.; Sevov, C. S. J. Am. Chem. Soc. 2020, 142, 5884.

(h) Perkins, R. J.; Pedro, D. J.; Hansen, E. C. Org. Lett. 2017, 19, 3755.

(i) Perkins, R. J.; Hughes, A. J.; Weix, D. J.; Hansen, E. C. Org. Process Res. Dev. 2019, 23, 1746.

[28] Jiang, W.-T.; Yang, S.; Xu, M.-Y.; Xie, X.-Y.; Xiao, B. Chem. Sci. 2020, $11,488$.

[29] (a) Wang, X.-X.; Yu, L.; Lu, X.; Zhang, Z.-L.; Liu, D.-G.; Tian, C.; Fu, Y. CCS Chem. 2021, 3, 727.

(b) Lu, X.; Xiao, B.; Zhang, Z.; Gong, T.; Su, W.; Yi, J.; Fu, Y.; Liu, L. Nat. Commun. 2016, 7, 11129.

(c) Wang, J.-W.; Li, Y.; Nie, W.; Chang, Z.; Yu, Z.-A.; Zhao, Y.-F.; $\mathrm{Lu}, \mathrm{X}$.; Fu, Y. Nat. Commun. 2021, 12, 1313.

[30] Supranovich, V. I.; Levin, V. V.; Kokorekin, V. A.; Dilman, A. D. Adv. Synth. Catal. 2021, 363, 2888.

(Cheng, F.) 